\title{
Local and Network Behavior of a Bistable Vibrational Energy Harvester Considering Periodic and Quasiperiodic Excitations
}

\author{
Anitha Karthikeyan \\ Ton Duc Thang University \\ Arthanari Ramesh \\ Chennai Institute of Technology \\ Irene Moroz \\ University of Oxford \\ Prakash Duraisamy \\ Chennai Institute of Technology \\ Karthikeyan Rajagopal ( $\nabla$ rkarthiekeyan@gmail.com ) \\ Chennai Institute of Technology https://orcid.org/0000-0003-2993-7182
}

\section{Research Article}

Keywords: energy harvesters, chaos, network, chimera

Posted Date: March 16th, 2021

DOI: https://doi.org/10.21203/rs.3.rs-277335/v1

License: (c) (1) This work is licensed under a Creative Commons Attribution 4.0 International License. Read Full License 


\title{
Local and network behavior of a bistable vibrational energy harvester considering periodic and quasiperiodic excitations
}

\author{
Anitha Karthikeyan ${ }^{\mathrm{a}}$, Arthanari Ramesh ${ }^{\mathrm{b}}$, Irene Moroz $^{\mathrm{c}}$, Prakash Duraisamy ${ }^{\mathrm{d}}$, Karthikeyan \\ Rajagopal $\mathrm{e}^{\mathrm{e}, *}$
}

${ }^{a}$ Nonlinear Systems and Applications, Faculty of Electrical and Electronics Engineering, Ton Duc Thang University, Ho Chi Minh City, Vietnam.

anitha.karthikeyan@tdtu.edu.vn

${ }^{\mathrm{b}}$ Center for Materials Research, Chennai Institute of Technology, India. ramesha@citchennai.net

${ }^{c}$ Mathematical Institute, University of Oxford, Andrew Wiles Building, Oxford, UK.

irene.Moroz@maths.ox.ac.uk

${ }^{\mathrm{d}, \mathrm{e}}$ Center for Nonlinear Systems, Chennai Institute of Technology, India.

akarthikeyan.rajagopal@ citchennai.net

fprakash.duraisamy@citchennai.net

*Corresponding author

\begin{abstract}
Vibrational energy harvesters can exhibit complex nonlinear behavior when exposed to external excitations. Depending on the number of stable equilibriums the energy harvesters are defined and analyzed. In this work we focus on the bistable energy harvester with two energy wells. Though there have been earlier discussions on such harvesters, all these works focus on periodic excitations. Hence, we are focusing our analysis on both periodic and quasiperiodic forced bistable energy harvester. Various dynamical properties are explored, and the bifurcation plots of the periodically excited harvester shows coexisting hidden attractors. To investigate the collective behavior of the harvesters, we mathematically constructed a two-dimensional lattice array of the harvesters. A non-local coupling is considered, and we could show the emergence of chimeras in the network. As discussed in the literature energy harvesters can be efficient if the chaotic regimes can be suppressed and hence we focus our discussion towards synchronizing the nodes in the network when they are not in their chaotic regimes. We could successfully define the conditions to achieve complete synchronization in both periodic and quasiperiodically excited harvesters.
\end{abstract}

Keywords: energy harvesters, chaos, network, chimera

\section{Introduction}

Wireless sensors and other low powered health monitoring devices have been widely used in industrial, medical, military, engineering and environmental health monitoring areas [1-3]. These devices need a continuous power supply with least replacement cost and long-life time service. Their power supply requirements are not fully addressed using the traditional batteries [4]. 
Vibration energy is readily available in the environment in the form of wind, ocean waves, human motion and mechanical vibration [5]. Among these, the mechanical energy is one of the most suitable one for structural health monitoring due to its availability [4]. As a result, energy harvesting from mechanical vibration is a promising means to replace conventional power sources.

Linear resonant energy harvesters have been used to exploit ambient vibration energy based on linear resonant vibration principle. They are effective only if the excitation power is concentrated in a narrow band of frequency, i.e. stationary excitation. But when the excitation frequency does not match the resonant frequency the harvesting efficiency reduced severely. Many researchers try to solve this issue by expanding the bandwidth of frequency using techniques such as frequency up-conversion, resonance tuning and deliberate introduction of nonlinearity [6-12]. Among these, inclusion of nonlinearity to harvesters attracted a lot of attention. Monostable and bistable harvesters found its significance due to various advantages under different excitation conditions and initial conditions. Monostable harvesters hold a simple configuration and has one stable equilibrium state. Compare to linear systems they are able to widen the frequency response range [8]. But their performance drops when they are subjected to real-world scenarios such as random excitation, and have similar output to linear harvesters when they are excited by white noise vibrations [13]. As an improvement to the drawback of monostable harvesters, researchers have focused on multistable harvesters. Bistable harvester has been explored widely [14-17]. It has two potential wells with a barrier in between. If the excitation amplitude is high enough the system will jump between the stable wells creating an interwell oscillations with high amplitude. The shape of the wells influences the response of the system. When the wells are shallow the bandwidth of the harvester increases, however the amplitude of the response is reduced. Compared to monostable harvesters, bistable shows improvement on harvesting, when the amplitude of the excitation is large enough to trigger an interwell oscillation $[18,19]$.

In addition to that there are only minimal literatures found for studying the network behaviors of Energy harvesters but practically, these to be work in a network to obtain a significant output. Since the study on synchronization and incoherent oscillations of a horde non-locally coupled oscillators [20], an interesting phenomenon is pronounced as "chimera states". The dynamics of chimera states and different topologies to handle chimera has been investigated vigorously in recent literatures [21-23]. Existence of chimera states and its control are studied with experimental evidence in some literatures [24-29]. Hence considering undeniable mutual effects of chimera states in dynamics of network and spatio-temporal nature, the intriguing characteristics of the system can be analyzed and it becomes fruitful test ground for energy efficiency also.

Motivated from the above discussion, in this paper we formulated a Bistable Energy Harvester $(\mathrm{BEH})$ supplied with higher order nonlinearity under quasi-periodic excitation. Stability analysis is carried out and presented in section 3. Bifurcation plots and corresponding Lyapunov spectrum are derived for different scenario in section 4 . The major contribution of this work lies on network dynamics of BEH under periodic and quasi-periodic excitations, the simulations are portrayed and interpreted in section 5. Finally, we provided concluding remarks and highlighted the significance of the present study.

\section{Mathematical Modeling}

In Figure. 1 configuration of a nonlinear energy harvester is shown. The configuration of bistable energy harvester consists of a stainless-steel substrate with two lead zirconate titanate (PZT) 
piezoelectric layers positioned near the base and two tip magnets. There are two external magnets located with required distance and angle.

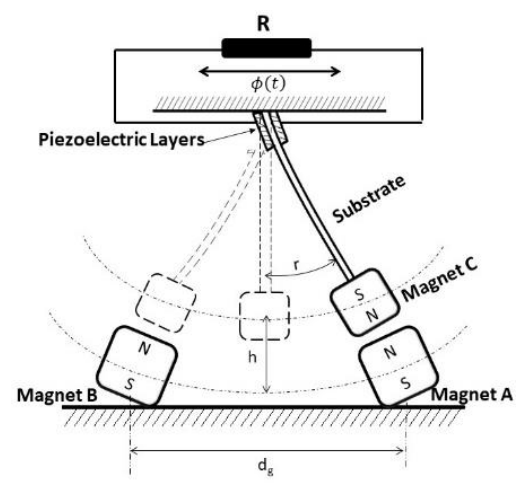

Fig 1. Configuration of bistable energy harvester (BEH)

The equation of motion for the BEH configuration presented in figure. 1 is derived as follows:

$$
\begin{aligned}
& m \&(t)+c \&(t)+F_{h}-\theta V(t)=\Phi(t) \\
& C_{p} L \&(t)+\frac{V(t)}{R}+\theta \& \&(t)=0
\end{aligned}
$$

Where,

$m, c$ are the equivalent mass and damping, $C_{p}$ refers equivalent capacitance, $\theta$ represents electromechanical coupling coefficient of the piezoelectric material, $R$ denotes load resistance. $V$ denotes the voltage, $r$ is the displacement and $\Phi(t)$ refers the external excitation.

For brevity, the equations of motion can be further nondimensionalized by using the following terms:

$$
x=\frac{r}{l_{c}}, t=t \omega_{n}, V=\frac{C_{p}}{\theta l_{c}} V
$$

Where, $l_{c}$ is a length scale introduced to nondimensionalize the displacement, $\omega_{n}=\sqrt{\frac{k}{m}}$ is the natural frequency of the harvester. With these transformations, the nondimensional model can be expressed as

$$
\begin{aligned}
& 2 \xi \&+F_{h}-\kappa^{2} V=\Phi(t) \\
& \&+\alpha V+\&=0
\end{aligned}
$$

Where,

$$
\xi=\frac{c}{2 \sqrt{k_{1} m}} \quad, \quad \delta=\frac{k_{3} l_{c}^{2}}{k_{1}}, \kappa=\frac{\theta^{2}}{k_{1} C_{c}} \quad, \quad \alpha=\frac{1}{\omega_{n} C_{p} R}
$$

here, $x$ represents displacement, $\omega_{n}$ is the natural frequency, and $\xi$ refers the damping ratio. In this paper we introduced a quartic nonlinearity term as restoring force, and the expression is as follows, 


$$
F_{h}=-x+\beta x^{2}+\delta x^{3}+\gamma x^{4}
$$

Most of the analysis done on existing models are with periodic excitation. Literatures on experimental studies of BEH shows that Quasi-periodic excitation affects the performance significantly, hence we are introducing quasi-periodic excitation. Generally quasi-periodic excitation will induce a strange Nonchaotic attractor (SNCA), and results with multistability. Hence in this paper we considered the system is supplied with quasi-periodic excitation and denoted as $\Phi(t)=A_{1}\left[\sin \left(\omega_{1} t\right)+A_{2} \sin \left(\omega_{2} t\right)\right]$.

The state space equation of the system can be written as,

$$
\begin{aligned}
& \&=y \\
& \&=A_{1}\left[\sin \left(\omega_{1} t\right)+A_{2} \sin \left(\omega_{2} t\right)\right]-2 \xi y+x-\delta x^{3}-\beta x^{2}-\gamma x^{4}+\kappa z \\
& \&=-y-\alpha z
\end{aligned}
$$

\section{Equilibrium points and stability analysis}

$F_{h}(x)$ is the nonlinear restoring force, while $\Phi(t)$ is the external excitation: Periodic if $A_{2}=0$ and quasi-periodic if $A_{2} \neq 0$. We take the parameter values in equ(5) to be $A_{1}=0.5, \omega_{1}=1$, $\omega_{2}=\frac{\sqrt{5}-1}{2}, A_{2}=1, \xi=0.0933, \delta=0.5495, \beta=0.1, \gamma=0.1, \alpha=0.4065, \kappa=0.00185$.

Stanton et al.,[30] performed a Melnikov analysis on a simplified bistable harvester by considering perturbations from a Hamiltonian limit. We follow part of their analysis here.

We re-write (5) as a perturbed Hamiltonian system:

$$
\left[\begin{array}{l}
\& \\
\& \\
\&
\end{array}\right]=\left[\begin{array}{c}
y \\
F_{h}(x) \\
0
\end{array}\right]-\varepsilon\left[\begin{array}{c}
0 \\
-2 \xi y+\kappa z+f(t) \\
-y-\alpha z
\end{array}\right]
$$

Where we have introduced a small parameter $\varepsilon$ to represent the non-Hamiltonian terms. When $\varepsilon=0$, eqn (6) becomes

$x=y$

$$
\&=x-\beta x^{2}-\delta x^{3}-\gamma x^{4}
$$

Which leads to the Hamiltonian

$$
E(t)=\frac{1}{2} y^{2}+V(x)
$$

With the potential energy function $V(x)$

$$
V(x)=-\frac{1}{2} x^{2}+\frac{\beta}{3} x^{3}+\frac{\delta}{4} x^{4}+\frac{\gamma}{5} x^{5}
$$

The fixed points of (7) satisfy $(x, y)=(0,0), y=0$ and the cubic roots of $1-\beta x-\delta x^{2}-\gamma x^{3}$. The trival fixed point $(0,0)$ is a saddle point. Values for the parameters, we find the remaining fixed points to be $x_{1}=-4.8675$ (another saddle point), $x_{2}=-1.7810$ and $x_{3}=1.1535$ (both centers). 


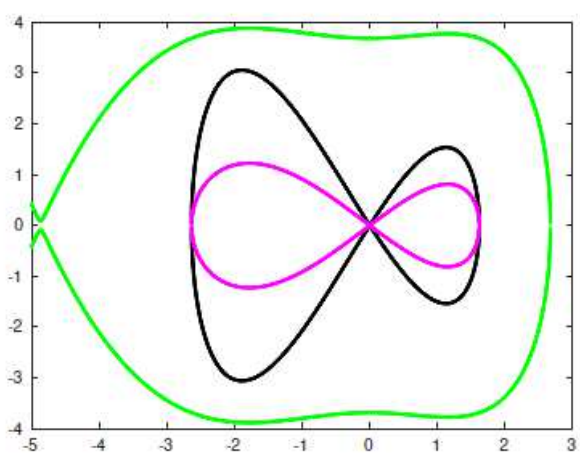

Fig 2: The double homoclinic loop for the BEH (magenta), passing through the fixed points $(0,0)$ and enclosing the two centres $x_{2}$ and $x_{3}$. There is also a large homoclinic loop (green), passing through $x_{1}$ and enclosing the other fixed points.

Fig.2 shows the double homoclinc loop in black refers to the simplified model with $\gamma=0$. homoclinic loop (magenta) that passes through the saddle point $(0,0)$, as well as the large homoclinic loop through the saddle point $x_{1}=-4.8675$. For the double homoclinic loop, $E(t)=0$ , while for the large homoclinic loop, $E(t)=6.781$. Solving (9) for y with $E(t)=0$ for the chosen parameter values, we get

$$
y_{D H}= \pm x[(x+5.8548)(x+2.6347)(1.6207-x)]
$$

So that the separatrix through the double homoclinic loop passes through $x s_{2}=1.6207$ and $x s_{1}=-2.6347$. There is also a separate branch (not shown) that passes through $x=-5.8548$, whose components tend to $\pm \infty$. Figure 2 shows the potential function $V(x)$, including the two end points $x s_{1}$ and $x s_{2}$ of the separatrix for the double homoclinic loop.

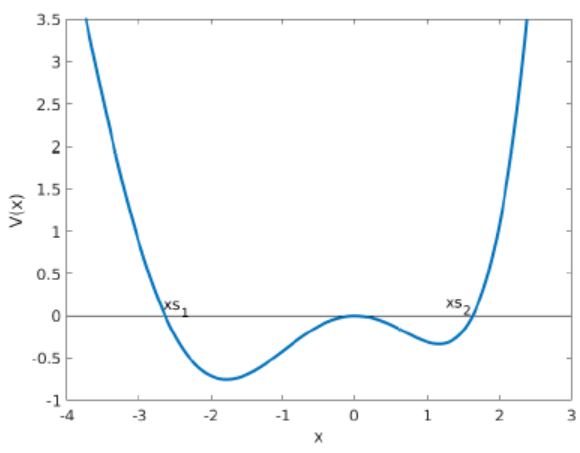

Fig 3: The potential function $V(x)$ for the BEH with the end points $x s_{1}$ and $x s_{2}$ of the separatrix of the double homoclinic loop

For the large homoclinic loop (green), we got $x=-4.8677$ (twice), $x=2.6711$ (the point at which the large orbit crosses the y-axis), and two other complex valued roots.

If we set $\gamma=0$, the model considered by Wang et al[31], we no longer get the root $x=-5.8548$, and the result is the larger black double homoclinic loop, shown in fig 2 . Since $E(t)=0$, eqns (10) and (11) give

$$
y=\frac{d x}{d t}= \pm x \sqrt{g(x)}
$$

Where 


$$
\begin{aligned}
g(x) & =a_{2} x^{2}+a_{1} x+a_{0} \\
& =1-\frac{2 \beta x}{3}-\frac{\delta x^{2}}{2} \\
& =-\left(x-x s_{1}\right)\left(x-x s_{2}\right)
\end{aligned}
$$

Litak and Borowiec [32] investigated the case of an asymmetric double homoclinic loop for a potential function of the form eqn(10) with $\gamma=0$. In eqn (15), we have taken $x s_{1}$ and $x s_{2}$ to be the end points on the black and magenta separatrices of figure 1, given in eqn(11). Equ (14) uses the expressions for $a_{j}$ from eqn (10). For (13) we obtain

$$
t=\int \frac{d x}{x \sqrt{g(x)}}=-\frac{1}{\sqrt{a_{0}}} \ln \left|\frac{2 a_{0}+a_{1} x+2 \sqrt{a_{0} g(x)}}{x}\right|
$$

Provided $a_{0}>0$. Since $a_{0}=0$ (by rearranging eqn(9), this is certainly the case here.

we can invert eqn (17) to find an expression for $x$ and then for $y$ for the double homoclinic loop. Defining $E=\exp t \sqrt{a_{0}}$, taking the exponential of both sides of (17) and rearranging gives, after straightforward algebra, $x=0$ (the saddle point at the origin) and

$$
x_{d h}=\frac{4 a_{0} E}{\left[\left(E-a_{1}\right)^{2}-4 a_{0} a_{2}\right]}
$$

The derivative $\frac{d x_{d h}}{d t}$ gives the y coordinate for the double homoclinic loop:

$$
y_{d h}=\frac{4 E a_{0}^{3 / 2}\left[a_{1}^{2}-4 a_{0} a_{2}-E^{2}\right]}{\left[\left(E-a_{1}\right)^{2}-4 a_{0} a_{2}\right]}
$$

\subsection{The unforced BEH system}

For the full BEH with $\gamma$ reinstated, we can find a solution for $x_{d h}$ in terms of Jacobi Elliptic functions of the first and third kinds, and involving inverse trigonometric sine functions. We omit their expressions here as not being very instructive. There is, also, no simple equation for the large green homoclinic loop, shown in Fig 2. Instead, some level sets, corresponding to potential function $V(x)$ for the full BEH system, are shown in Fig 3.

In the absence of external forcing (so that $\Phi(t)=0$ ), the equilibrium states are given by $y=0, z=0$ and $x$.s a solution to $F_{h}(x)=0$. We therefore obtain the trivial equilibrium $x=0$, together with the three nontrivial equilibrium states obtained in the Hamiltonian limit, namely a saddle point and two centres. For the given set of parameter values, these are the roots of the RHS of eqn (1.7). The linear stability of the equilibrium state is determined by the eigen spectrum of the characteristic equation:

$$
\lambda^{3}+\Delta_{2} \lambda^{2}+\Delta_{1} \lambda+\Delta_{0}=0
$$




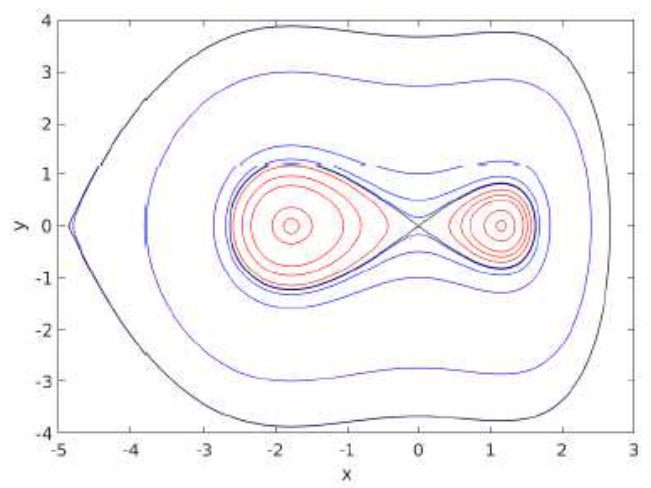

Fig 4: Some level sets for the potential function V(x) for the BEH. The separatrix for the double homoclinic loop and the large homoclinic loop are shown as black curves.

Where,

$$
\begin{aligned}
& \Delta_{2}=2 \xi+\alpha \\
& \Delta_{1}=2 \xi \alpha+\kappa-G_{x} \\
& \Delta_{0}=-\alpha G_{x} \\
& G_{x}=1-2 \beta x-3 \delta x^{2}-4 \gamma x^{3}
\end{aligned}
$$

For the trivial equilibrium state $x=0, G_{x}=\alpha$. This means there are no steady bifurcations from the trivial equilibrium. Moreover, by substituting $\lambda=i \omega$ into eqn (19), it is straightforward to show that there are also no possible Hopf bifurcations, since this would require $\Delta_{2}+\alpha=0$ : not possible when all parameters are positive. Codimension one steady state bifurcations can occur when $\Delta_{0}=0$ for the nontrivial saddle point at $\gamma=0.139$ (keeping all remaining parameter values at their prescribed values). The condition for a Hopf bifurcation, $\Delta_{0}-\Delta_{1} \Delta_{2}=0$, is not possible since this would require

$$
\omega^{2}=\frac{\alpha G_{x}}{2 \xi+\alpha}=2 \xi \alpha+\kappa-G_{x}
$$

Numerical integrations show that these two criteria for $\omega^{2}$ cannot be simultaneously satisfied.

Moreover, numerical integrations for the unforced system also show that the only stable states are steady states; we found no evidence of periodic solutions. When the $z$-dependence is present, the saddle point $x_{1}$ remains a saddle, while the two centers $x_{2}$ and $x_{3}$ become stable foci. For the parameter values $\xi=0.0933 ; \delta=0.5495 ; \alpha=0.4065 ; \beta=0.1 ; \gamma=0.1 ; \kappa=0.00184$ the equilibrium points and corresponding eigen values are calculated and presented in table. 1 for understanding the stability.

Table 1. Equilibrium points and its Stability

\begin{tabular}{|c|c|c|}
\hline Equilibrium Points & Eigen Values & Stability \\
\hline$E_{0}=[0 ; 0 ; 0]$ & {$[0.9104 ;-1.0962 ;-0.4073]$} & Saddle node \\
\hline$E_{1}=[-4.8675 ; 0 ; 0]$ & {$[2.9155 ;-3.1020 ;-0.4066]$} & Saddle node \\
\hline$E_{2}=[-1.781 ; 0 ; 0]$ & {$[-0.0935 ; \pm 1.2673 i ;-0.4061]$} & Stable focus \\
\hline
\end{tabular}




$$
\begin{array}{|l|l|l|}
E_{3}=[1.1535 ; 0 ; 0] & {[-0.0935 ; \pm 1.4252 i ;-0.4061]} & \text { Stable focus } \\
\hline
\end{array}
$$

The system shows a chaotic attractor for the following parameter values, $A_{1}=0.5, \omega_{1}=1, A_{2}=1$, $\omega_{2}=\frac{\sqrt{5}-1}{2}, \xi=0.0933, \delta=0.5495, \beta=0.1, \gamma=0.1, \kappa=0.00184, \alpha=0.4065$ for the initial condition $\{1,0,1\}$. The 2D Phase portraits are given in Fig 5.

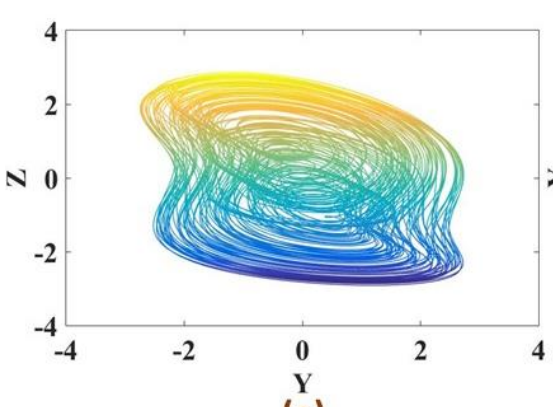

(a)

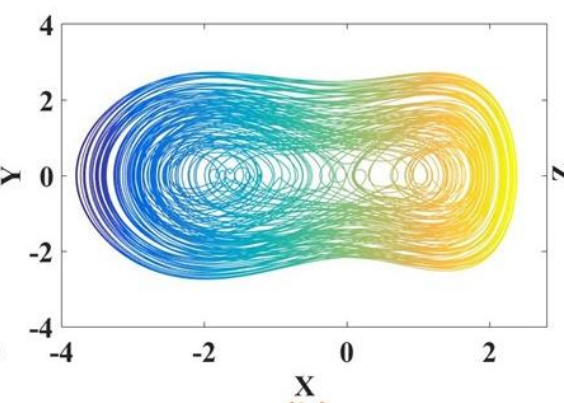

(b)

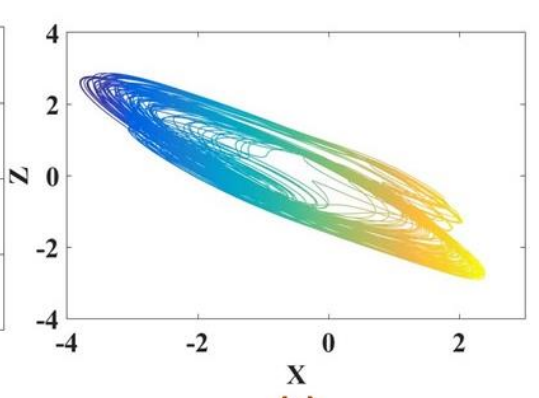

(c)

Fig 5. $2 D$ Phase portrait of system (6) for the parameter values $A_{1}=0.5, \omega_{1}=1, A_{2}=1, \omega_{2}=\frac{\sqrt{5}-1}{2}$, $\xi=0.0933, \delta=0.5495, \beta=0.1, \gamma=0.1, \kappa=0.00184, \alpha=0.4065$ and initial condition $\{1,0,1\}$

\section{Bifurcation and Lyapunov Spectrum}

We investigated the bifurcation property for two scenario Case (1) the system under periodic excitation, Case (2) the system under Quasi-Periodic excitation. We used Runge-Kutta numerical method for simulating the results. We considered the parameter values: $A_{1}=0.5, A_{2}=1, \xi=0.0933$ $, \delta=0.5495, \quad \beta=0.1, \gamma=0.1, \kappa=0.00184, \alpha=0.4065$ and initial condition $\{1,0,1\}$. For investigating Bi-stability nature of the system under mentioned excitation conditions, we provided the bifurcation plots using forward continuation (Plotted with blue dots), backward continuation (Plotted with Red dot) and the corresponding Lyapunov spectrum is calculated using Wolf algorithm [33] and plotted for a finite time of $20000 \mathrm{~s}$.

\section{Case (1). Under periodic excitation}

Firstly, we considered the system (5) is supplied with periodic excitation $\Phi(t)=F \sin (\omega t)$ and the response of the system is noted and presented as bifurcation plot. In Fig. 6 we could observe the bistability phenomena which is considered dangerous for mechanical systems. We varied the parameter $\omega$ for the range of 0.9 to 1.3. For detailed analysis we provided the bistable regions in Fig. 6(b) and the corresponding Lyapunov spectrum for forward and backward continuation also presented in Fig 6(c) \& 6(d). 

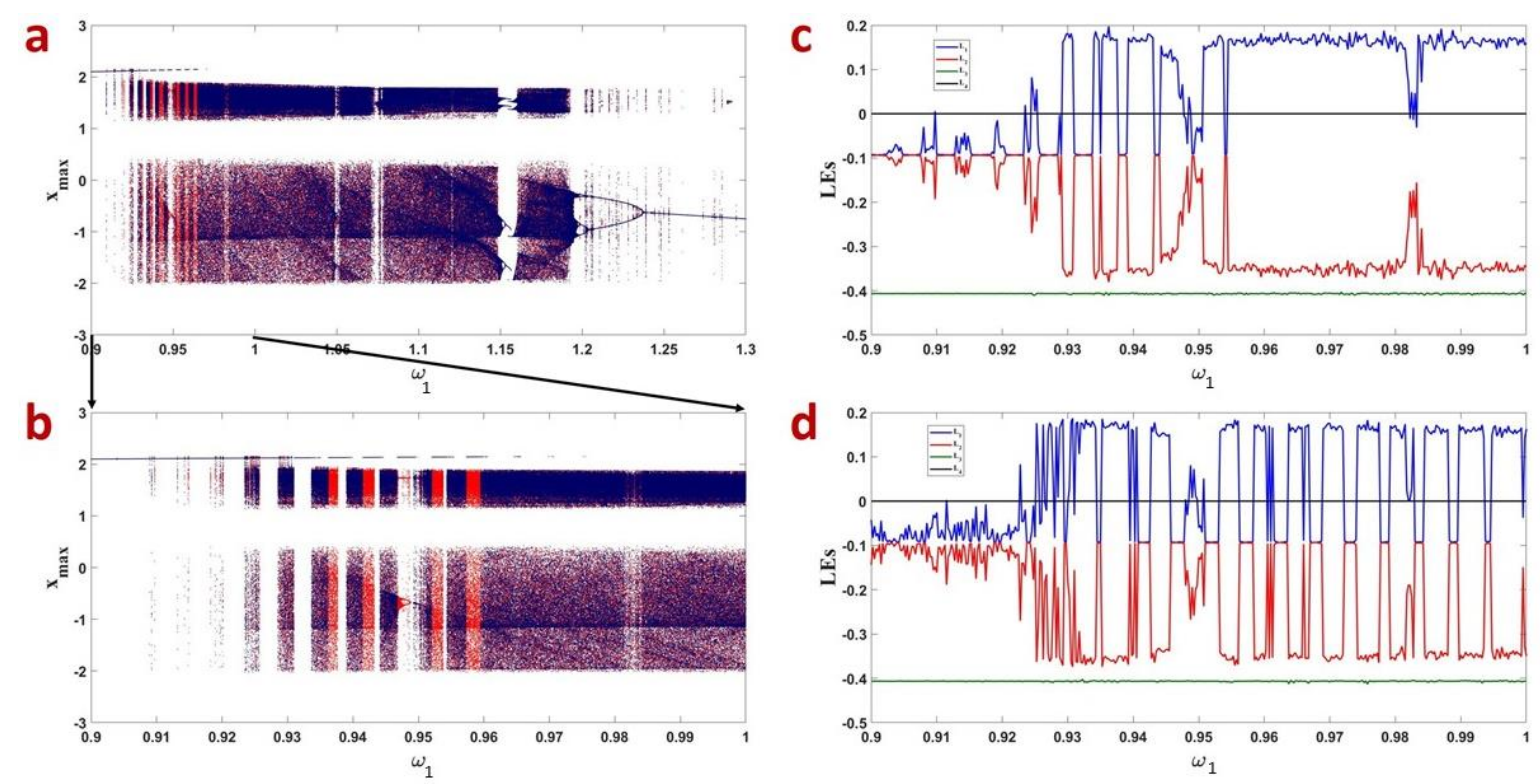

Fig 6. Bifurcation plot and corresponding Lyapunov Exponents of parameter $\omega_{1}$ variation. (Periodic excitation)

\section{Case (2). Under Quasi-periodic excitation}

The system (5) is supplied with higher order nonlinear stiffness function under quasi-periodic excitation; hence the influence of excitation frequency plays vital role in behavioral analysis. In this section, we derived the bifurcation plots for frequency range $0 \leq \omega_{1} \leq 3$. The corresponding Lyapunov Spectrum also presented. We could observe that the multistability property is vanished during quasi-periodic excitation.
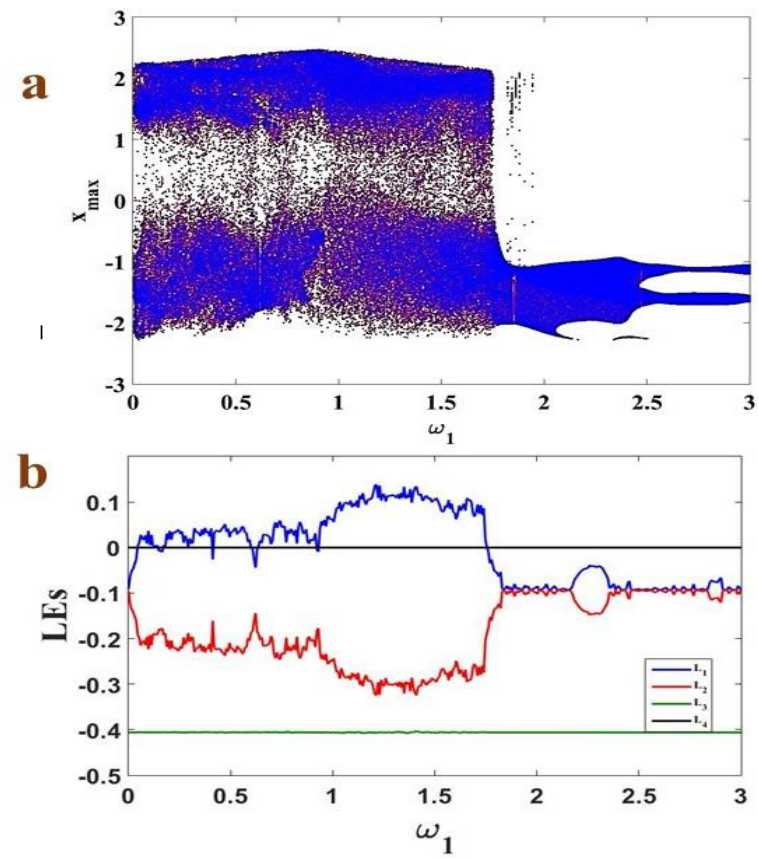
Fig 7. Bifurcation plot and corresponding Lyapunov Exponents of parameter $\omega$ variation (Quasi-Periodic excitation)

\section{Network dynamics of BEH:}

The local behaviour of the BEH with periodic and quasi-periodic excitations shows some interesting dynamical behaviours but energy harvesters will normally be applied in large networks. Hence, we consider $\mathrm{N}$ coupled BEH whose mathematical model is shown in (25),

$$
\begin{aligned}
& \underset{i}{\&}=y_{i}+\sigma \sum_{j=1}^{N} C_{i j} x_{j} \\
& \underset{t}{\&}=\Phi(t)-2 \xi y_{i}+x_{i}-\delta x_{i}^{3}-\beta x_{i}^{2}-\gamma x_{i}^{4}+\kappa z_{i} \\
& \underset{j}{\&}=-y_{i}-\alpha z_{i}
\end{aligned}
$$

The term $\sigma$ defines the coupling constant and the connection between the nearby nodes are defined by the connection matrix $C_{i j}$. The external excitation is defined by $\Phi(t)=A_{1}\left[\sin \left(\omega_{1} t\right)+A_{2} \sin \left(\omega_{2} t\right)\right]$ where $\omega_{1}=1$ is the frequency of the periodic term while $\omega_{2}=\frac{\sqrt{5}-1}{2}$ is the golden mean contributing to the quasi-periodic excitation. We have considered two different cases for discussion depending on the type of excitation applied to the nodes in the network.

\subsection{Network behaviour with periodic excitation}

In this case we consider $A_{2}=0$, we now apply a periodic excitation to the nodes in the network and for simulation we consider the parameters as $A_{1}=0.5, \omega_{1}=1, \xi=0.0933, \delta=0.5495$, $\beta=0.1, \gamma=0.1, \kappa=0.00184, \alpha=0.4065$ and random initial conditions are chosen. We have used RK4 method to solve the system (25) with the step size of 0.01 and total simulation time of 3000s. In Fig. 8 we have shown the spatiotemporal behaviour of the network for coupling strengths $\sigma \leq 0.015$ for which we could see that the nodes are in complete incoherency. We have also plotted the instantaneous state variable value of $x$ measured at the end of simulation. For $\sigma=$ 0.015 the nodes try to achieve synchronisation and leads us to a clue to check for chimeras by increasing coupling. 

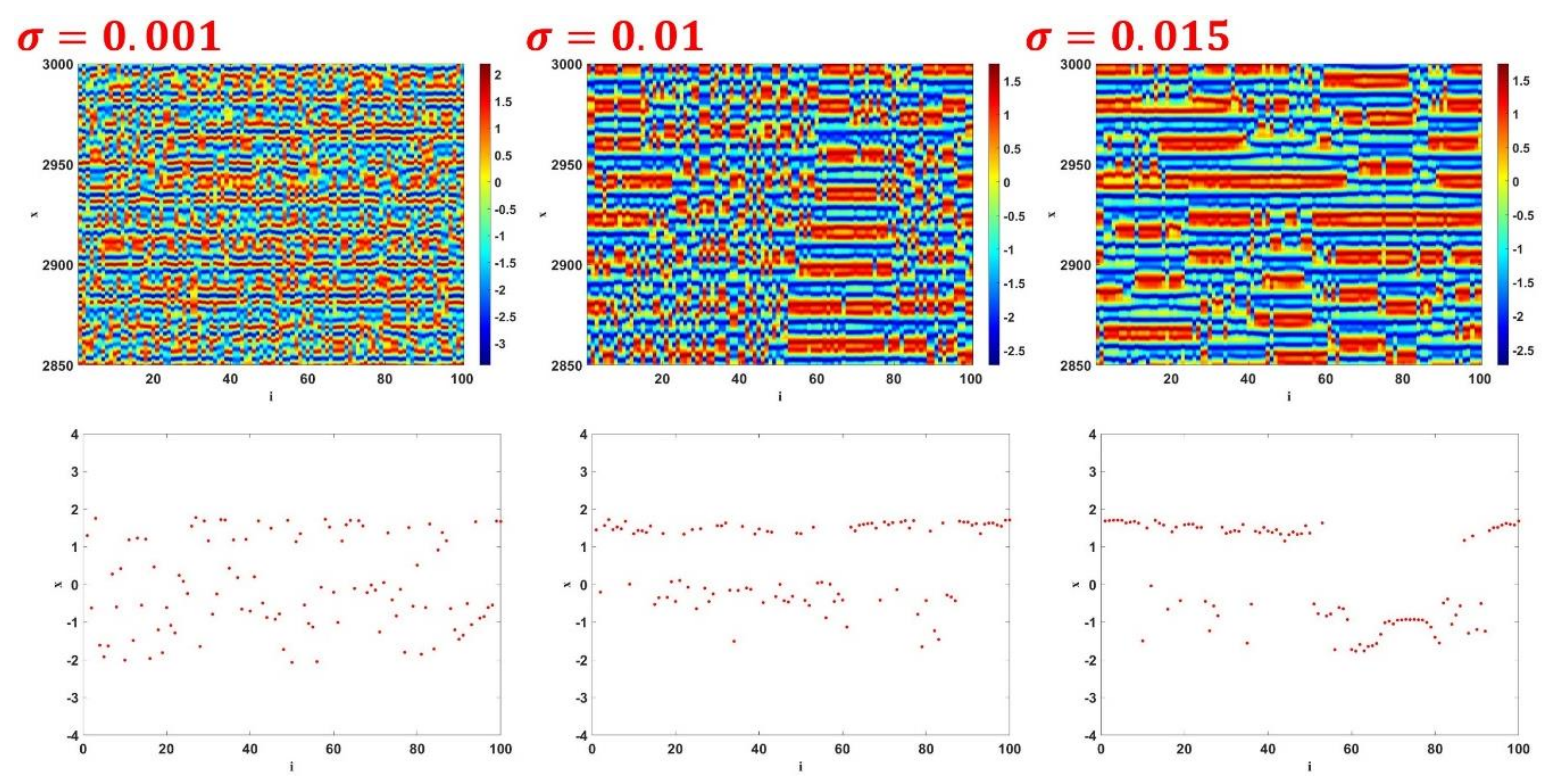

Fig.8: The collective behaviour of the network (1) for different values of the coupling coefficient ( $\sigma$ ) considering a periodic excitation. The plots confirm that the nodes are asynchronous.

Now we increased the coupling to $\sigma=0.018$ to identify the existence of coherent and incoherent nodes in the network. In Fig.9 we could see that most of the nodes try to achieve coherency, some nodes are still in complete incoherent state. This confirms the existence of chimeras in the network. It should be noted that only if all the BEH nodes are in coherent state, the energy efficiency will be more, and such chimeras will result in residue current in the BEH nodes which could damage the node permanently. Such chimeras are seen in the network for $\sigma<0.03$ but we have only shown spatiotemporal behaviour of selected values of $\sigma$.

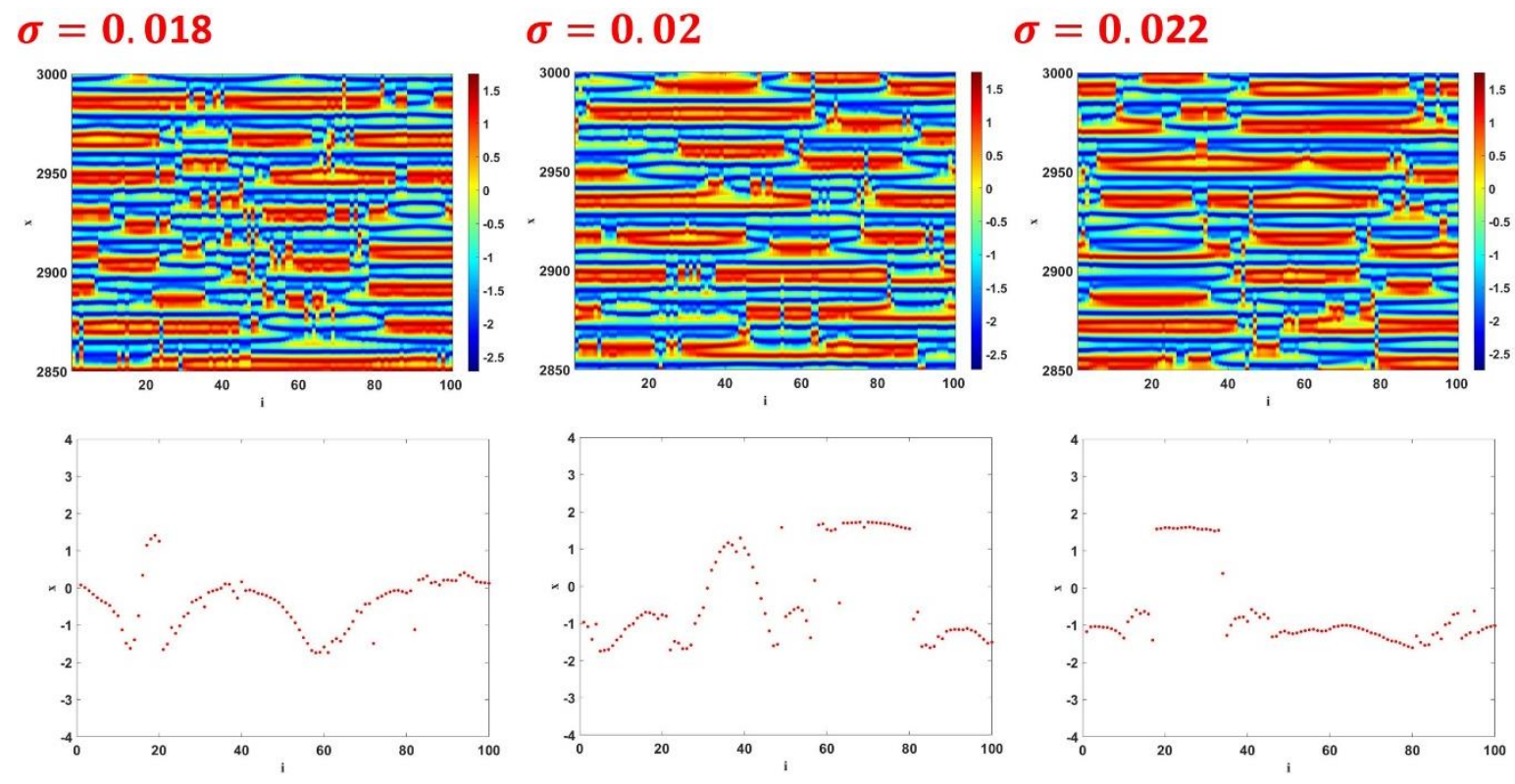

Fig.9 The collective behaviour of the network (25) for different values of the coupling coefficient ( $\sigma$ ) considering a periodic excitation. The plots confirm that the nodes are showing both synchronous and asynchronous nodes confirming the existence of chimeras. 
Further increasing the coupling strength to $\sigma=0.03$, the nodes try to achieve synchronisation in small clusters. Though such phenomenon of cluster synchronisation is not uncommon in spiking networks, its undesirable in such networks where complete synchronisation is mandatory. In other words, we need all the BEH nodes in the network to operate in a single frequency or at least in coherency to maximise the energy efficiency of the harvesters. In Fig. 10 we have shown several cluster synchronisations conditions for various values of $\sigma$.
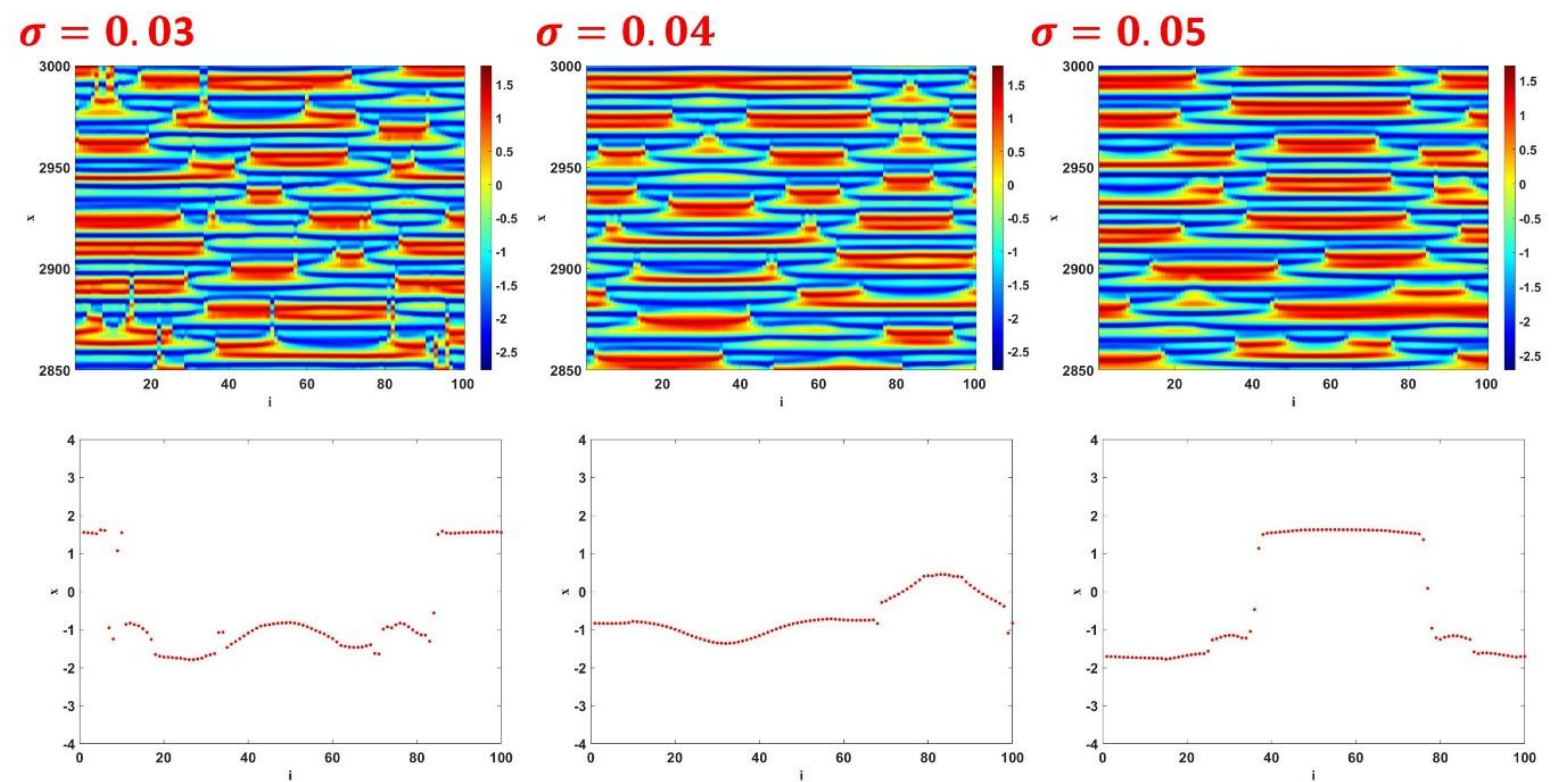

Fig.10: The collective behaviour of the network (25) for different values of the coupling coefficient $(\sigma)$ considering a periodic excitation. The plots confirm several clusters of synchronised BEH nodes in the network.

We have used re-occurrence plots to identify different regimes of synchronisation in the network. The re-occurrence plots are calculated by finding the Euclidean distance between $x_{i}$ and $x_{j}$ where $i . j \in[1, N]$. In Fig. 11 we have shown the re-occurrence plots and the absence of structures in the plot for $\sigma=0.001$ shows that there are no coherent nodes in the network. For $\sigma=0.01$, we could see small structures formed in the network confirming the emergence of chimeras. For the remaining values of $\sigma<0.03$ shown in the plots we could see majority of blue and red regions. The blue region shows coherent oscillators and the red shows the incoherent oscillators. The presence of other colours in these plots confirm the existence of multiple intermediate nodes which neither belong to the red and nor to the blue regions confirming different incoherent frequencies in the network. But when $\sigma>0.03$ we could note only blue and red dominant confirming different clusters of synchronisations. 

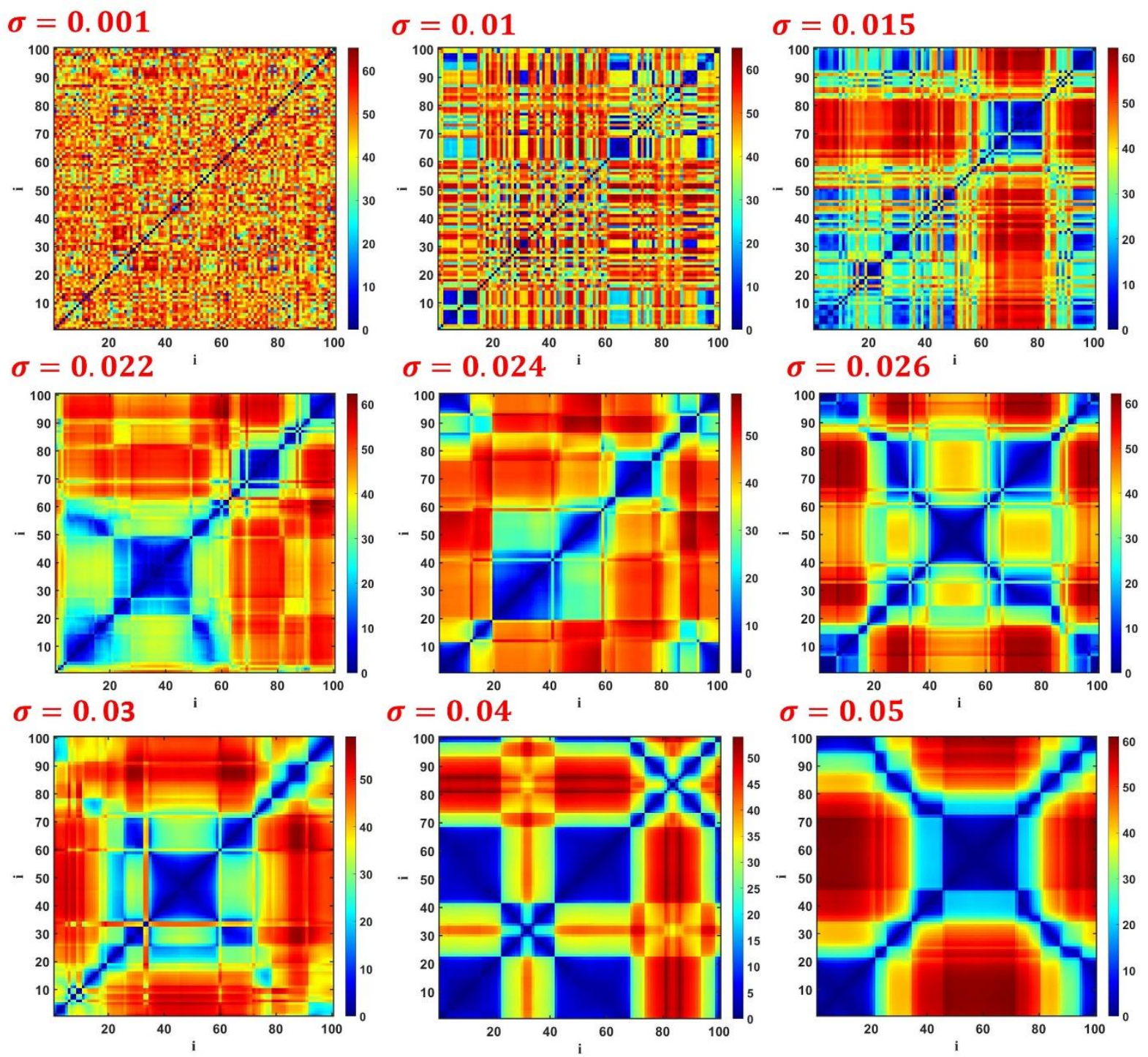

Fig.11: Re-occurrence plots of the BEH network for different values of the coupling coefficient. The blue regions show the coherent oscillators and the red show the incoherent oscillators.

Though we could achieve cluster synchronisation in the network as shown in Fig.11, we couldn't reach complete synchronisation. For values of coupling $\sigma>0.1$, the network goes in to unbounded states and thus we couldn't use the coupling strength to achieve synchronisation. As the BEH are externally excited by the periodic force, our interest now is on the amplitude of the periodic force which can be properly tuned to achieve complete synchronisation. In Fig. 12 we have shown the spatiotemporal plots for the amplitude values $A_{1}=[0.4,0.45,0.5]$ and the plots confirm the emergence of chimeras in the network. Though for $A_{1}=0.5$, the incoherent nodes are very less confirming that the nodes are moving to complete synchronization. 


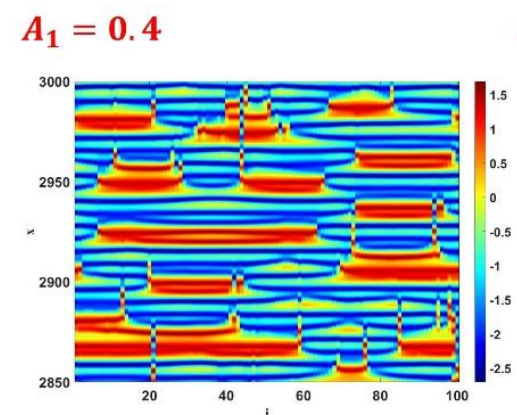

$$
A_{1}=0.45
$$
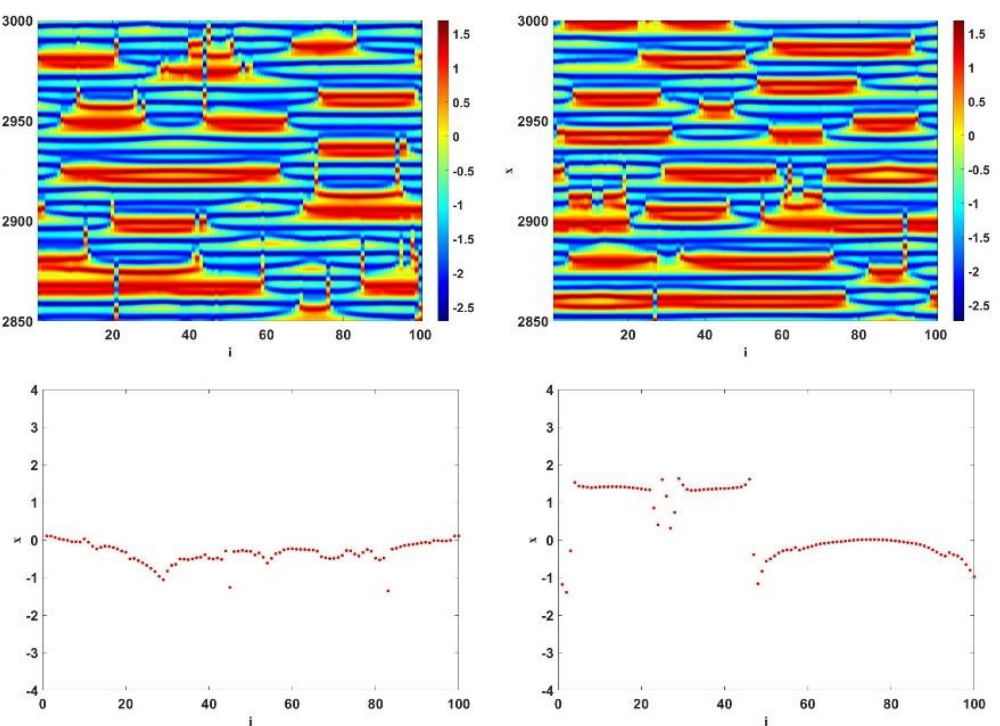

$A_{1}=0.5$
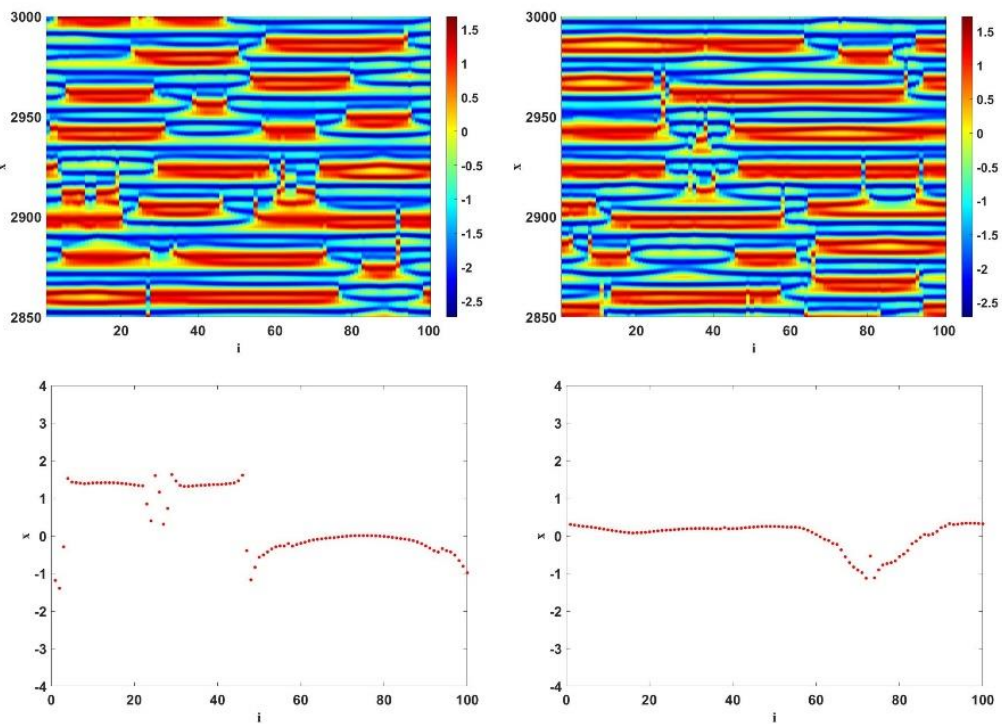

Fig.12 The collective behaviour of the network (1) for different values of the of the amplitude of the periodic excitation considering $\sigma=0.024$. The plots emergence of chimera states with synchronous and asynchronous nodes

In Fig.13, we have shown the complete synchronisation of the nodes achieved through the tuning of the amplitude of the external excitation. We could note that for $A_{1}<0.4$ and $A_{1}>0.6$ the nodes attain complete synchronisation and achieves the same frequency of operation. Thus, we could achieve maximum efficiency from the energy harvesters when connected in a network. But its been shown in the literatures that non-chaotic BEH [34] can be productive in energy harvesting but as seen from the re-occurrence plot (Fig.12 right most) for $A_{1}=0.3$, the nodes are no more chaotic and shows periodic behaviours and for $A_{1}=0.7$ the nodes are chaotic and are completely synchronised as there Euclidean distance is very low in $10^{-13}$. Thus, we recommend an amplitude value of $A_{1}=0.3$ to achieve complete synchronisation in the periodically excited BEH network. 

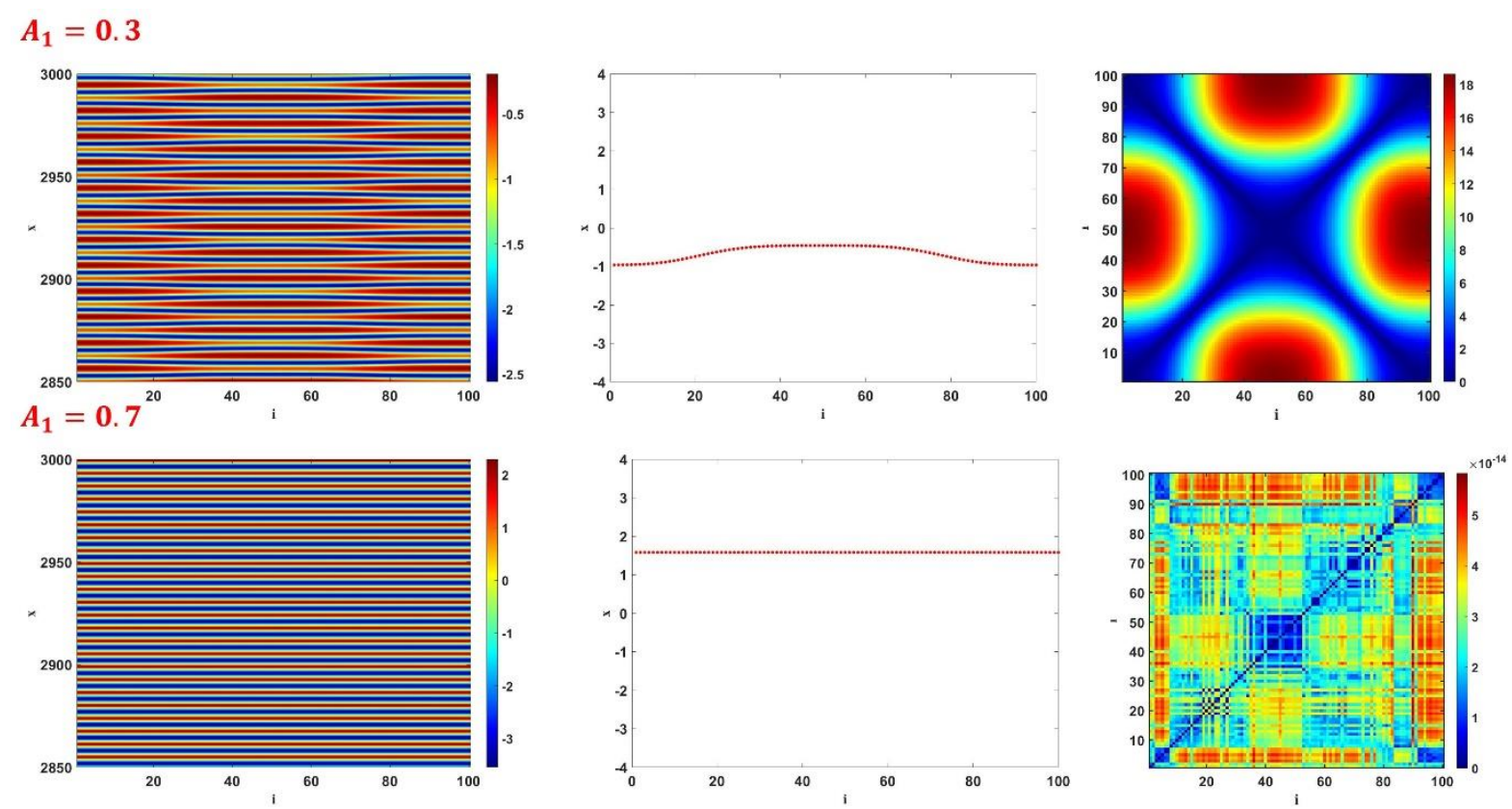

Fig.13: The collective behaviour of the network (25) for different values of the of the amplitude of the periodic excitation considering $\sigma=0.024$. The plots confirm the nodes achieving complete synchronisation.

\subsection{Network behaviour with quasi-periodic excitation}

In this case we consider $A_{1}=0.5$, we now apply a quasi-periodic excitation to the nodes in the network by considering $A_{2} \neq 0, \omega_{2}=\frac{\sqrt{5}-1}{2}$ with the other parameters as $\omega_{1}=1, A_{2}=1$, $\xi=0.0933, \delta=0.5495, \beta=0.1, \gamma=0.1, \kappa=0.00184, \alpha=0.4065$. The other setting for simulations is similar to section 5.1. As we have earlier shown that the coupling coefficient couldn't be tuned to achieve complete synchronisation (section 5.1), we verified the same for quasi and could again confirm that $\sigma$ cannot be tuned for synchronisation in quasi-periodic case. Hence, we haven't provided the discussion on $\sigma$ as it will be redundant. We focus our discussion on the amplitude of the quasi-periodic term $\left(A_{2}\right)$ and have kept $\sigma=0.025$. In Fig.14 we have shown the spatiotemporal plots for different values of $A_{2}$ and unlike Fig.13, we couldn't find periodic regimes for $A_{2}<1$ and the network shows both coherent and incoherent nodes confirming the existence of chimeras. 
$A_{2}=0.1$
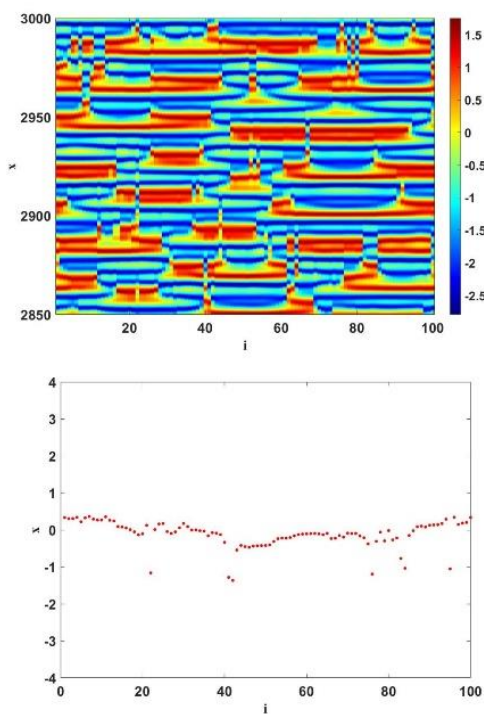

$A_{2}=0.5$
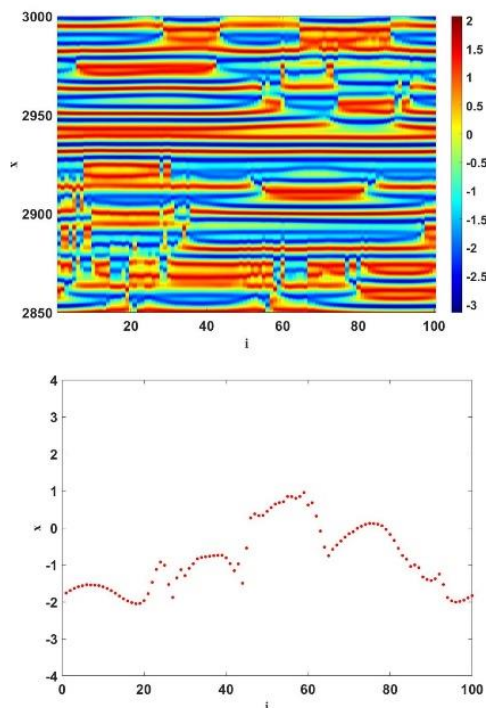

$A_{2}=0.8$
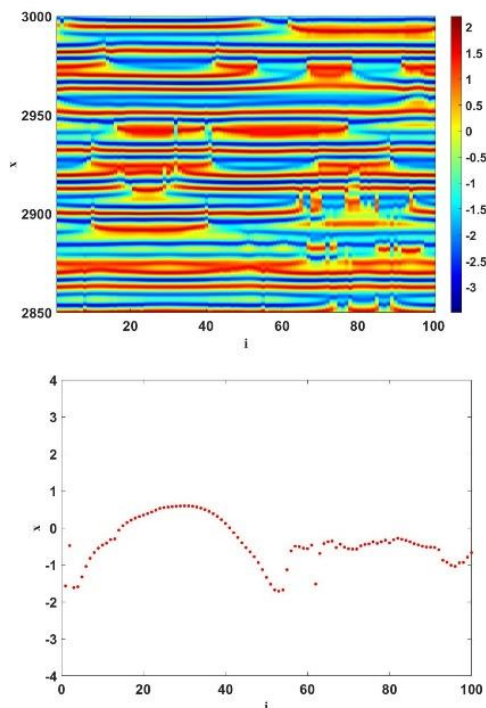

Fig.14: The collective behaviour of the network (25) for different values of the of the amplitude of the quasiperiodic excitation considering $\sigma=0.025$. The plots confirm the emergence of chimeras.

To find the amplitude values which could help us achieve complete synchronisation with nodes are not in their chaotic regime, we further increase the value of $A_{2}=1.5$ and could observe complete synchronisation and the same is the case for $A_{2}=1.8$, presented in Fig. 15. But our interest is to check whether the nodes are not in their chaotic regime while achieving complete synchronisation.

$A_{2}=1$
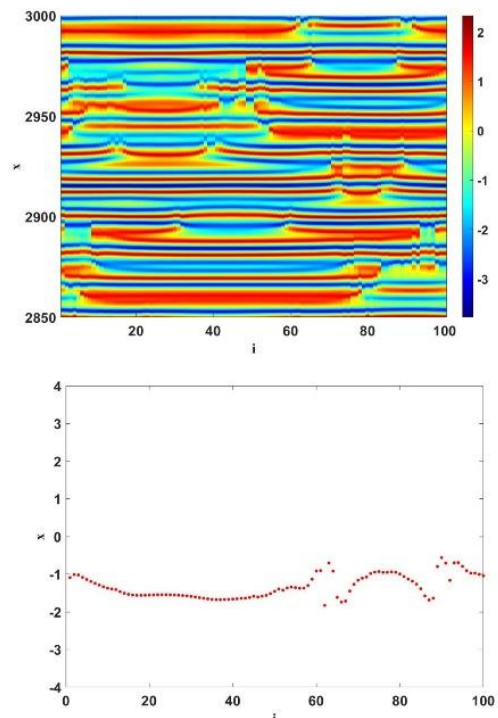

$$
A_{2}=1.5
$$
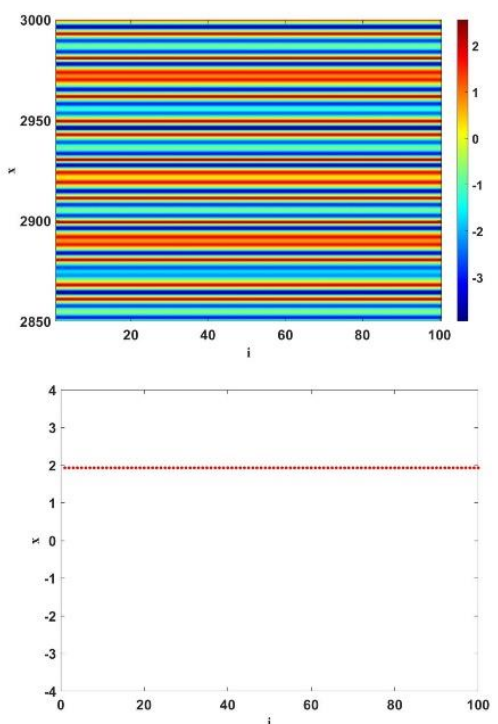

$$
A_{2}=1.8
$$
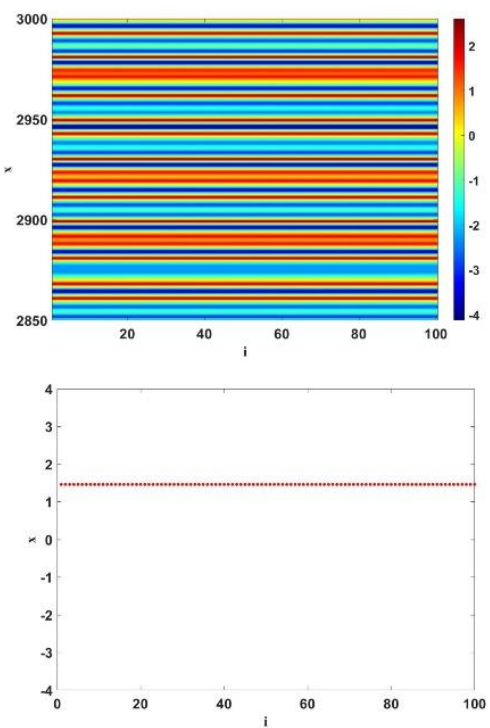

Fig.15: The collective behaviour of the network (25) for different values of the of the amplitude of the quasiperiodic excitation considering $\sigma=0.025$. The plots confirm nodes achieving complete synchronisation for $A_{2}=1.5$ and $A_{2}=1.8$ 
As discussed, we must verify which of the amplitude $\left(A_{2}\right)$ can achieve complete synchronisation while the BEH nodes are not chaotic. Hence, we use the re-occurrence plots as shown in Fig.16. While checking the re-occurrence plots for $A_{2}=1.5$ we could see that the nodes are in synchronisation with period-4 oscillations and for $A_{2}=1.8$, the nodes whose different colours confirming they are chaotic but their Euclidian distance in the range of $10^{-13}$ showing complete synchronisation. Hence when exposed to quasi-periodic excitation we could recommend the amplitude combination as $A_{1}=0.5$ and $A_{2}=1.5$ for complete synchronisation as for these two amplitude values the nodes are periodic and chaotic oscillations are supressed.
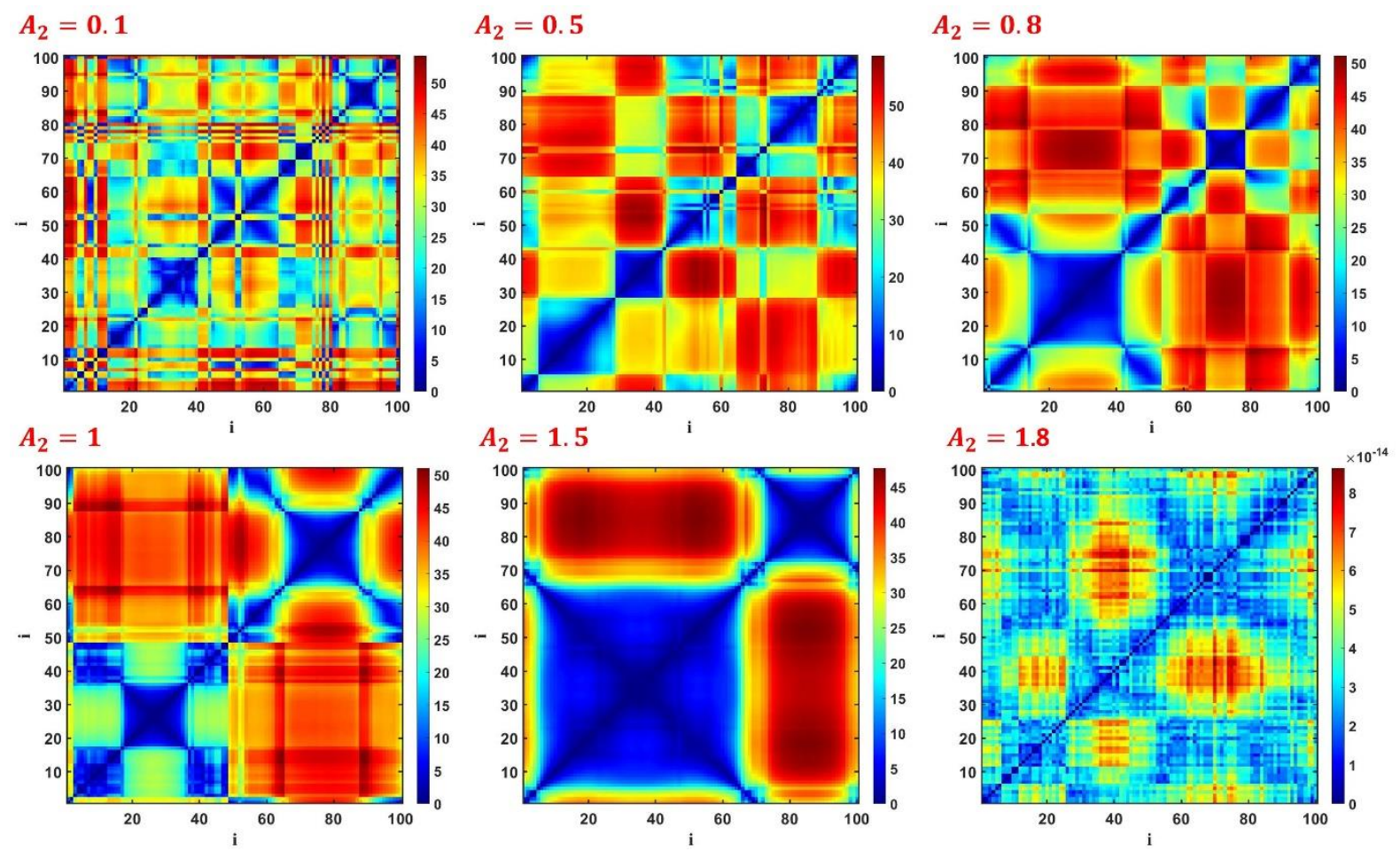

Fig.16: The re-occurrence plots for different values of $A_{2}$ with the blue regions showing coherent nodes while the other colours showing incoherent nodes.

\section{Conclusion}

Energy harvester model with two stable equilibrium points is analyzed considering periodic and quasiperiodic external excitations. The dynamical properties of the model is analyzed and we could show coexisting hidden attractors in the system for periodic excitation. Though local behaviour can help us understand the complex oscillations and bifurcation patterns of the bistable energy harvesters, our focus is to investigate its collective dynamics. A mathematical model of a 2D lattice network is constructed whose local dynamics is governed by the bistable energy harvesters. Firstly a periodic excitation is applied to the nodes and the spatiotemporal behavior is captured. We could observe regions of asynchronous nodes for very low coupling values while increasing the coupling some nodes are synchronized while some remain incoherent. This confirms the emergence of chimeras. We tried different values of the coupling to observe complete synchronization, but we could observe only cluster synchronization. Hence, we shifted our investigation to the excitation amplitude, and we could observe two ranges of amplitude which could achieve complete synchronization. But our interest is on the amplitude for which the nodes are not in chaos, but the 
network achieved synchronization. To calculate this, we introduced the re-occurrence plots which is useful in understanding different regimes in a network. From the re-occurrence plots we could show that for a certain amplitude the nodes are not in chaos, but the network achieved synchronization. Similar studies are conducted for quasiperiodically excited energy harvesters and again we could show some values of the amplitude can achieve synchronization without disturbing the local complex behavior of the nodes.

Conflict of Interest: The authors declare that they have no conflict of interest.

Funding: Arthanari Ramesh, Karthikeyan Rajagopal and Prakash Duraisamy have been partially funded by the Research grant of Center for Nonlinear Systems, Chennai Institute of Technology with reference number CIT/CNS/2021/RP-017.

\section{References}

[1] J. M. R. A. Erturk, D. J. Inman, "Modeling of Piezoelectric Energy Harvesting from an Lshaped Beam-mass Structure with an Application to UAVs," Journal of Intelligent Material Systems and Structures, vol. 20, pp. 529-544, 2009.

[2] C. E. S. C. A. Raghavan, "The Shock and Vibration Digest Review of Guided-wave Structural Health Monitoring," Shock Vib. Dig., vol. 39, pp. 91-114, 2007.

[3] F. B. J. M. Gilbert, "Comparison of energy harvesting systems for wireless sensor networks," International Journal of Automation and computing, vol. 5, pp. 334-347, 2008.

[4] R. M. Mohammed F. Daqaq, Alper Erturk, D. Dane Quinn, "On the Role of Nonlinearities in Vibratory Energy Harvesting: A Critical Review and Discussion," Appl. Mech. Rev., vol. 66, p. 040801, 2014.

[5] M. J. T. S. P. Beeby, N. M. White, "Energy harvesting vibration sources for microsystems applications," Measurement Science and Technology, vol. 17, p. 175, 2006.

[6] S. E. K. M. Rezaei, P. Firoozy, "Broadband and tunable PZT energy harvesting utilizing local nonlinearity and tip mass effects," International Journal of Engineering Science, vol. 118, pp. 1-15, 2017.

[7] J. C. S. Zhou, J. Lin, "Theoretical analysis and experimental verification for improving energy harvesting performance of nonlinear monostable energy harvesters," Nonlinear Dynamics, 2016.

[8] Q. T. K. Fan, Y. Zhang, S. Liu, M. Cai, Y. Zhu, "A monostable piezoelectric energy harvester for broadband low-level excitations," Appl. Phys. Lett., 2018.

[9] M. G. P. V. R. Challa, Y. Shi, F. T. Fisher, "A vibration energy harvesting device with bidirectional resonance frequency tunability," Smart Mater. Struct., vol. 17, p. 15035, 2008.

[10] H. L. D. R. Naseer, A. Abdelkefi, L. Wang, "Piezomagnetoelastic energy harvesting from vortex-induced vibrations using monostable characteristics," Appl. Energy, vol. 203, pp. 142-153, 2017.

[11] C. L. W. Liu, X. Li, Q. Zhu, G. Hu, "Comparative study about the cantilever generators with different curve fixtures," 29, vol. 9, pp. 1884-1899, 2018.

[12] S. G. B. A. Cammarano, D. A. W. Barton, A. Carrella, L. R. Clare, "Tuning a resonant energy harvester using a generalized electrical load," Smart Mater. Struct., vol. 19, 2010. 
[13] S. P. Beeby et al., "A comparison of power output from linear and nonlinear kinetic energy harvesters using real vibration data," Smart Mater. Struct., vol. 22, p. 75022, 2013.

[14] X. Y. Li et al., "Piezoelectric material based energy generator using bistable cantilever beam," in Future Wireless Networks and Information Systems. vol. 143, Z. Y., Ed., ed Berlin, Heidelberg: Springer, 2012.

[15] B. A. O. B. P. Mann, "Investigations of a nonlinear energy harvester with a bistable potential well," J. Sound Vib., vol. 329, pp. 1215-1226, 2010.

[16] M. F. D. R. Masana, "Energy harvesting in the super-harmonic frequency region of a twinwell oscillator," Journal of Applied Physics, vol. 111, p. 044501, 2012.

[17] H. J. J. I.H. Kim, B.M. Lee, S.J. Jang, "Broadband energy-harvesting using a two degreeof-freedom vibrating body," Appl. Phys. Lett., vol. 98, p. 214102, 2011.

[18] M. F. D. M. Panyam, "A comparative performance analysis of electrically optimized nonlinear energy harvesters," Journal of Intelligent Material Systems and Structures, vol. 27, pp. 537-548, 2015.

[19] M. F. D. R. Masana, "Relative performance of a vibratory energy harvester in mono- and bi-stable potentials," J. Sound Vib., , vol. 330, pp. 6036-6052, 2011.

[20] Y. K. a. D. Battogtokh, "Coexistence of Coherence and Incoherence in Nonlocally Coupled Phase Oscillators," Nonlinear Phenomena in Complex Systems, vol. 4, pp. 380-385, 2002.

[21] L. M. P. D. M. Abrams, A. E. Motter, "Introduction to focus issue: Patterns of network synchronization," Chaos, vol. 26, p. 094601, 2016.

[22] D. M. A. M. J. Panaggio, "Chimera states: Coexistence of coherence and incoherence in networks of coupled oscillators," Nonlinearity, vol. 28, 2015.

[23] D. M. A. a. S. H. Strogatz, "Chimera states for coupled oscillators," Phys. Rev. Lett., vol. 93, p. 174102, 2004.

[24] A. B. L. V. Gambuzza, S. Chessari, L. Fortuna, R. Meucci, and M. Frasca, "Experimental investigation of chimera states with quiescent and synchronous domains in coupled electronic oscillators," Phys. Rev. E vol. 90, p. 032905, 2014.

[25] S. T. E. A. Martens, A. Fourrie`re, O. Hallatschek, "Chimera states in mechanical oscillator networks," Proc. Natl. Acad. Sci. , vol. 110, pp. 10563-10567, 2013.

[26] B. P. L. Larger, Y. Maistrenko, "Virtual chimera states for delayed-feedback systems," Phys. Rev. Lett. , vol. 111, p. 054103, 2013.

[27] I. Z. K. M. Wickramasinghe, "Spatially organized dynamical states in chemical oscillator networks: Synchronization, dynamical differentiation, and chimera patterns," PloS One vol. 8, p. e80586, 2013.

[28] S. N. M. R. Tinsley, K. Showalter, "Chimera and phase-cluster states in populations of coupled chemical oscillators," Nat. Phys, vol. 8, pp. 662-665, 2012.

[29] T. E. M. A. M. Hagerstrom, R. Roy, P. H€ovel, I. Omelchenko, E. Sch€oll, "Experimental observation of chimeras in coupled-map lattices," Nat. Phys, vol. 8, pp. 658-661, 2012.

[30] C. C. M. S. C. Stanton, B. P. Mann, "Nonlinear dynamics for broadband energy harvesting: Investigation of a bistable piezoelectric inertial generator," Physica D, vol. 239, pp. 640$653,2010$.

[31] J. C. W. Wang, C.R. Bowen, D. J. Inman, J. Lin, "Performance enhancement of nonlinear asymmetric bistable energy harvesting from harmonic, random and human motion excitations," Appl. Phys. Lett., vol. 112, p. 213903, 2018.

[32] G. Litak, Borowiec, M. , "Oscillators with asymmetric single and double well potentials: transition to chaos revisited," Acta Mechanica vol. 184, pp. 47-59, 2006. 
[33] A. Wolf, Swift, J.B., Swinney, H.L., Vastano, J.A., "Determining Lyapunov exponents from a time series," Physica D: Nonlinear Phenomena, vol. 16, pp. 285-317, 1985.

[34] Daqaq, M.F., Crespo, R.S. \& Ha, S. On the efficacy of charging a battery using a chaotic energy harvester. Nonlinear Dyn 99, 1525-1537 (2020). 
Figures

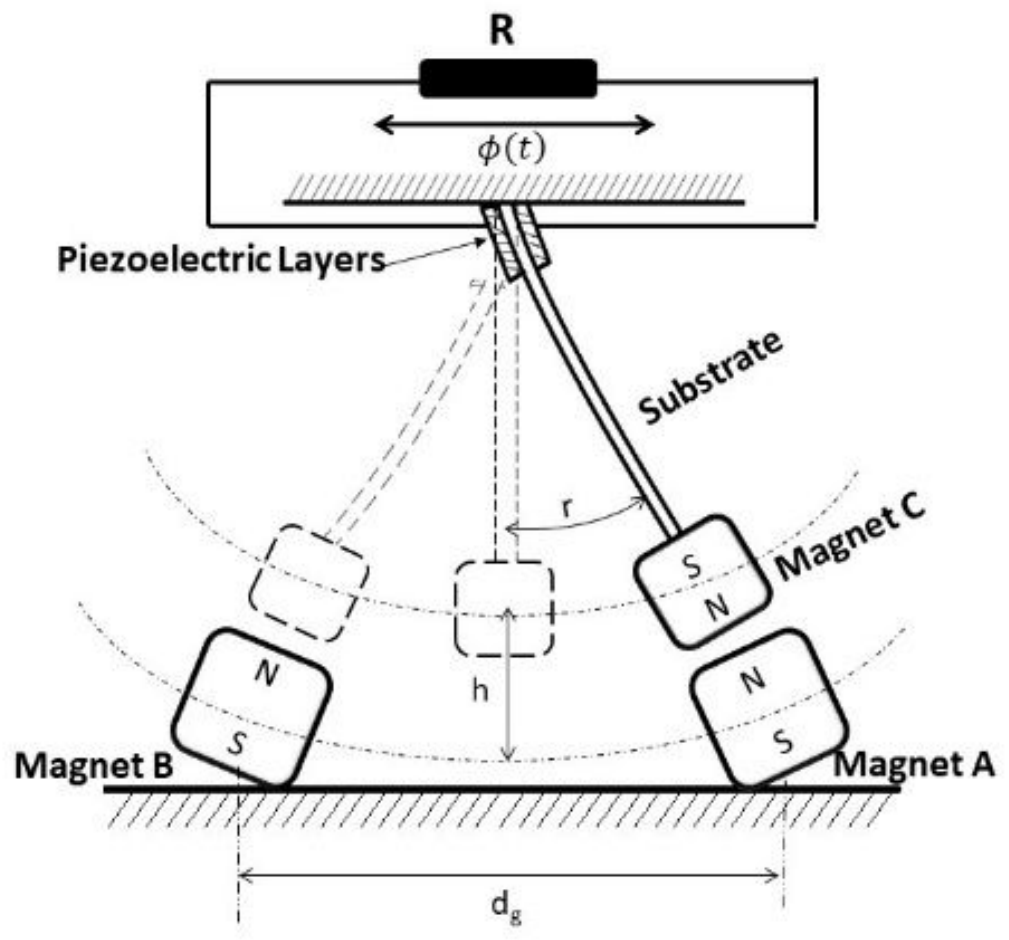

Figure 1

Configuration of bistable energy harvester (BEH)

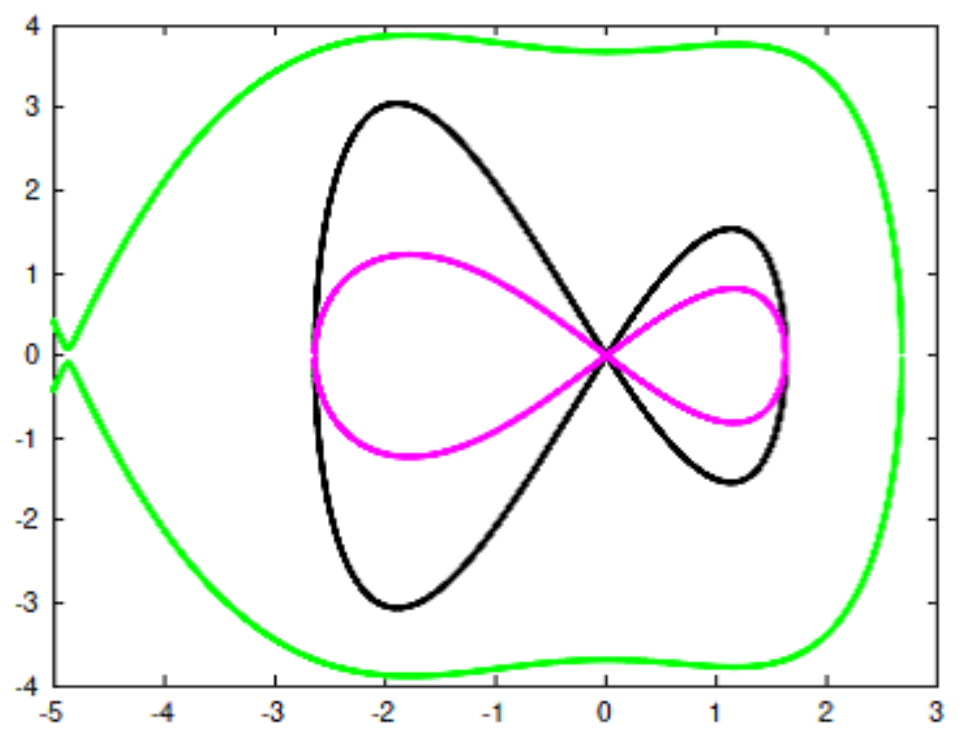

Figure 2

Please see the Manuscript PDF file for the complete figure caption 


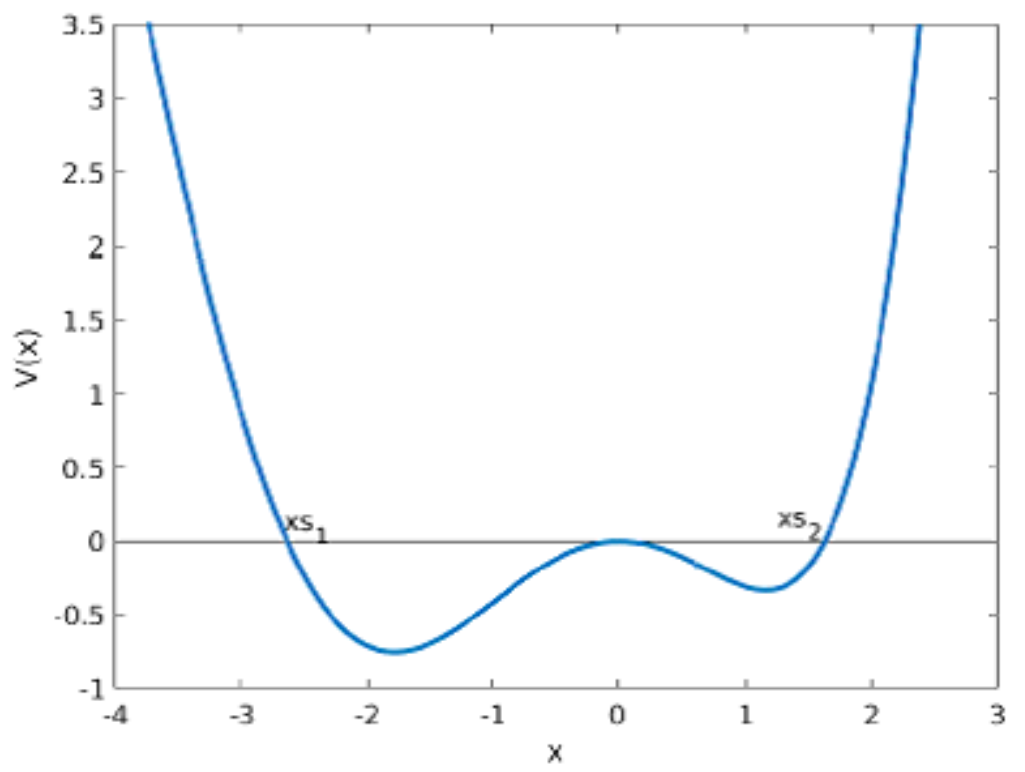

Figure 3

Please see the Manuscript PDF file for the complete figure caption

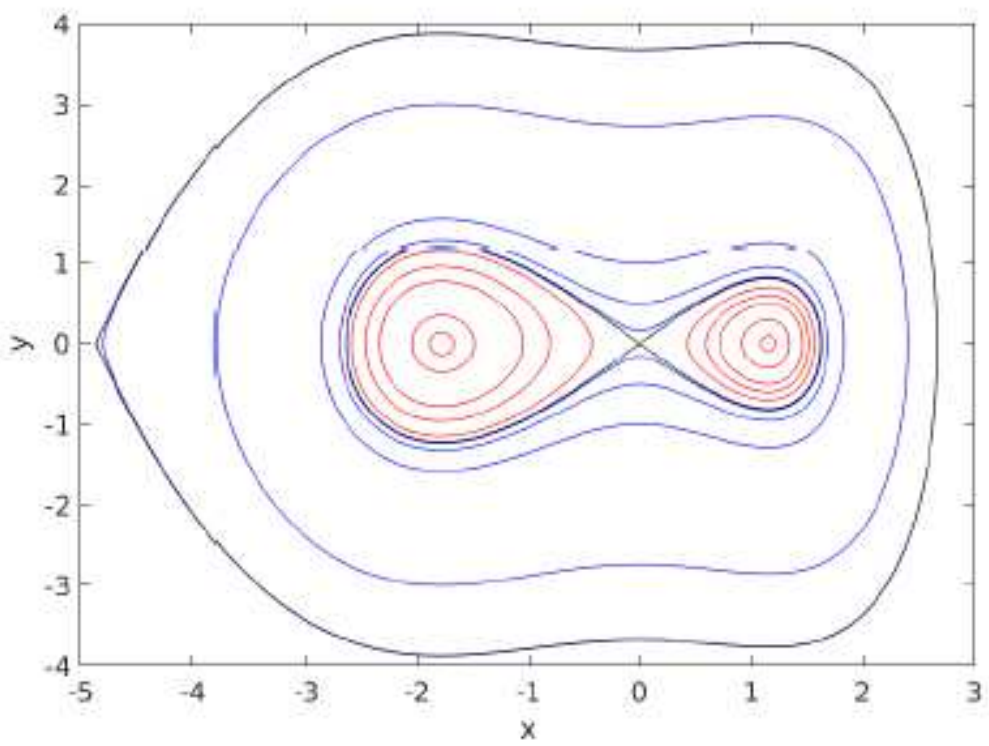

Figure 4

Please see the Manuscript PDF file for the complete figure caption 


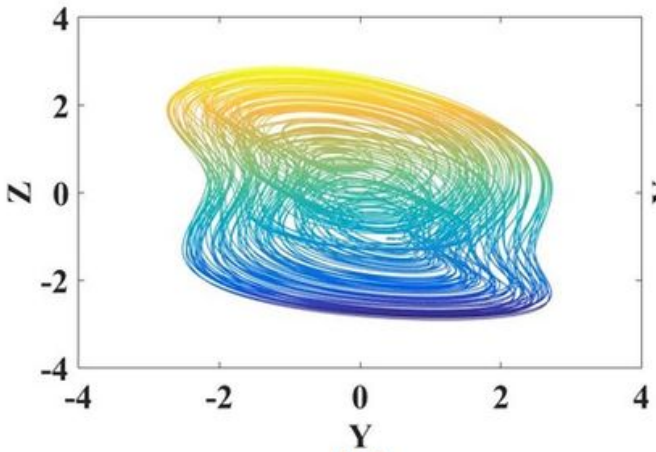

(a)

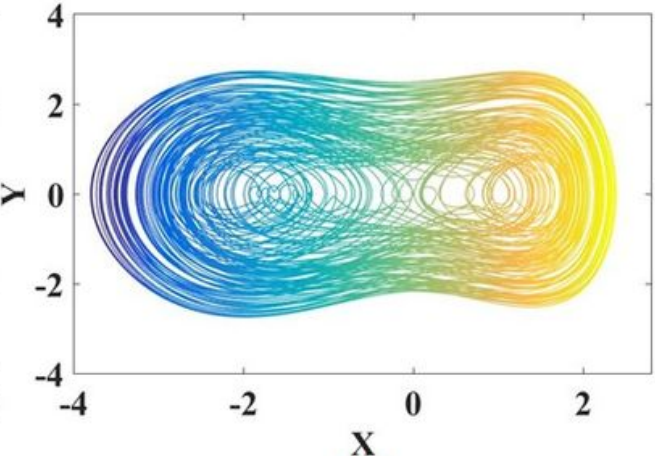

(b)

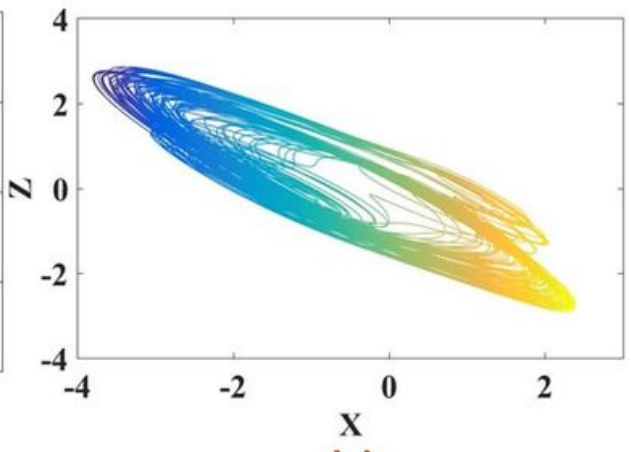

(c)

Figure 5

Please see the Manuscript PDF file for the complete figure caption
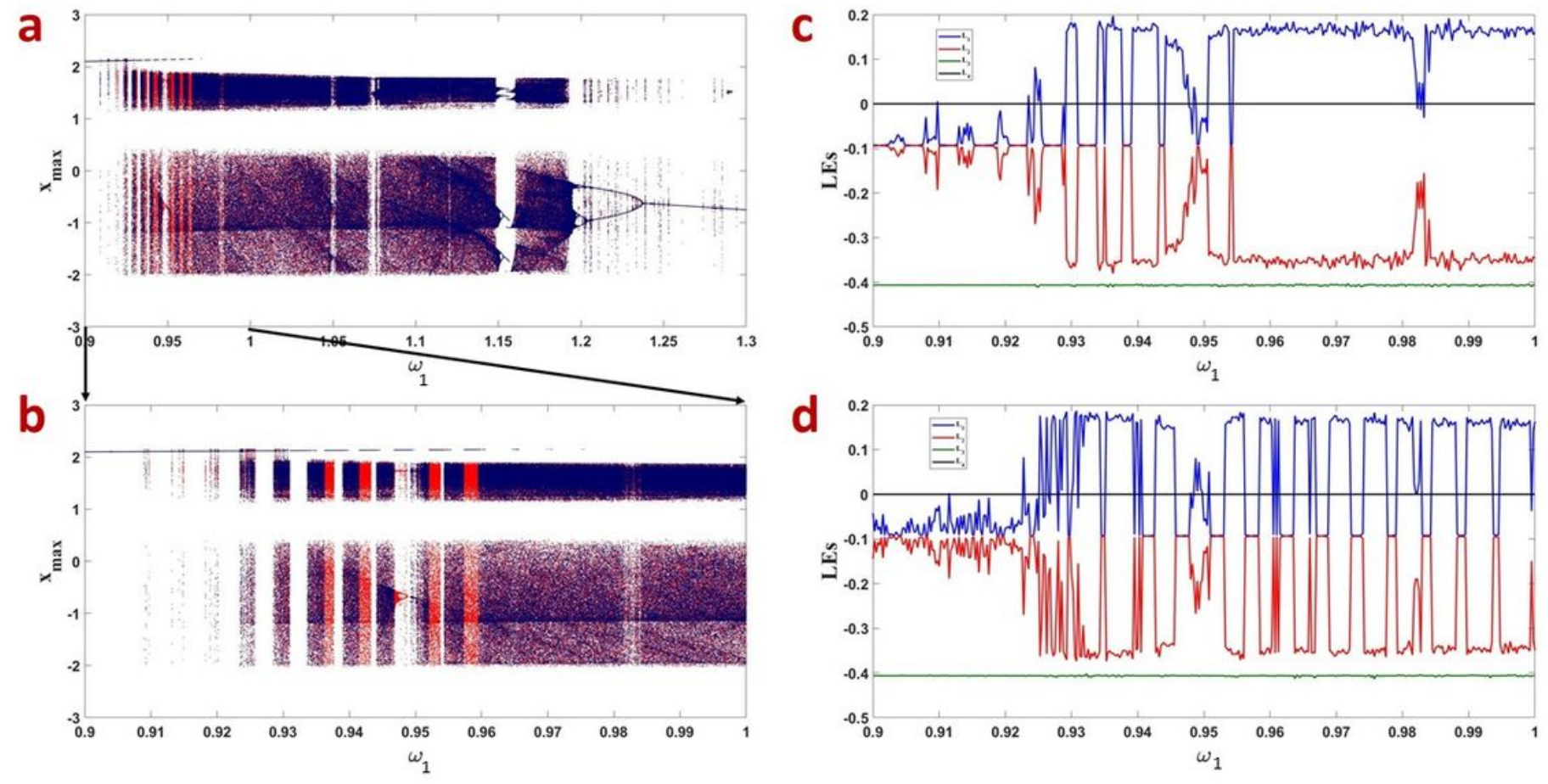
Figure 6

Please see the Manuscript PDF file for the complete figure caption
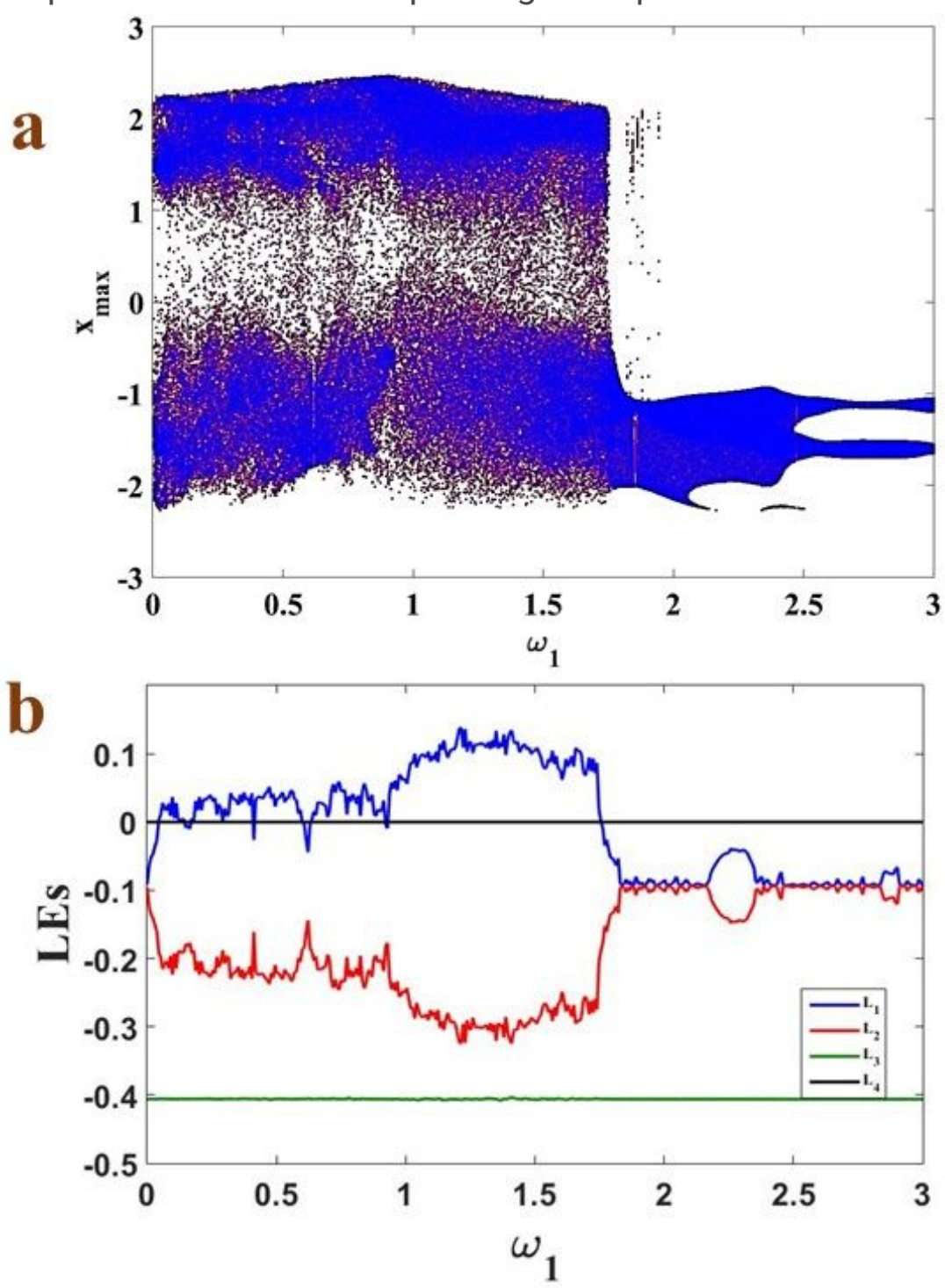

Figure 7

Please see the Manuscript PDF file for the complete figure caption 

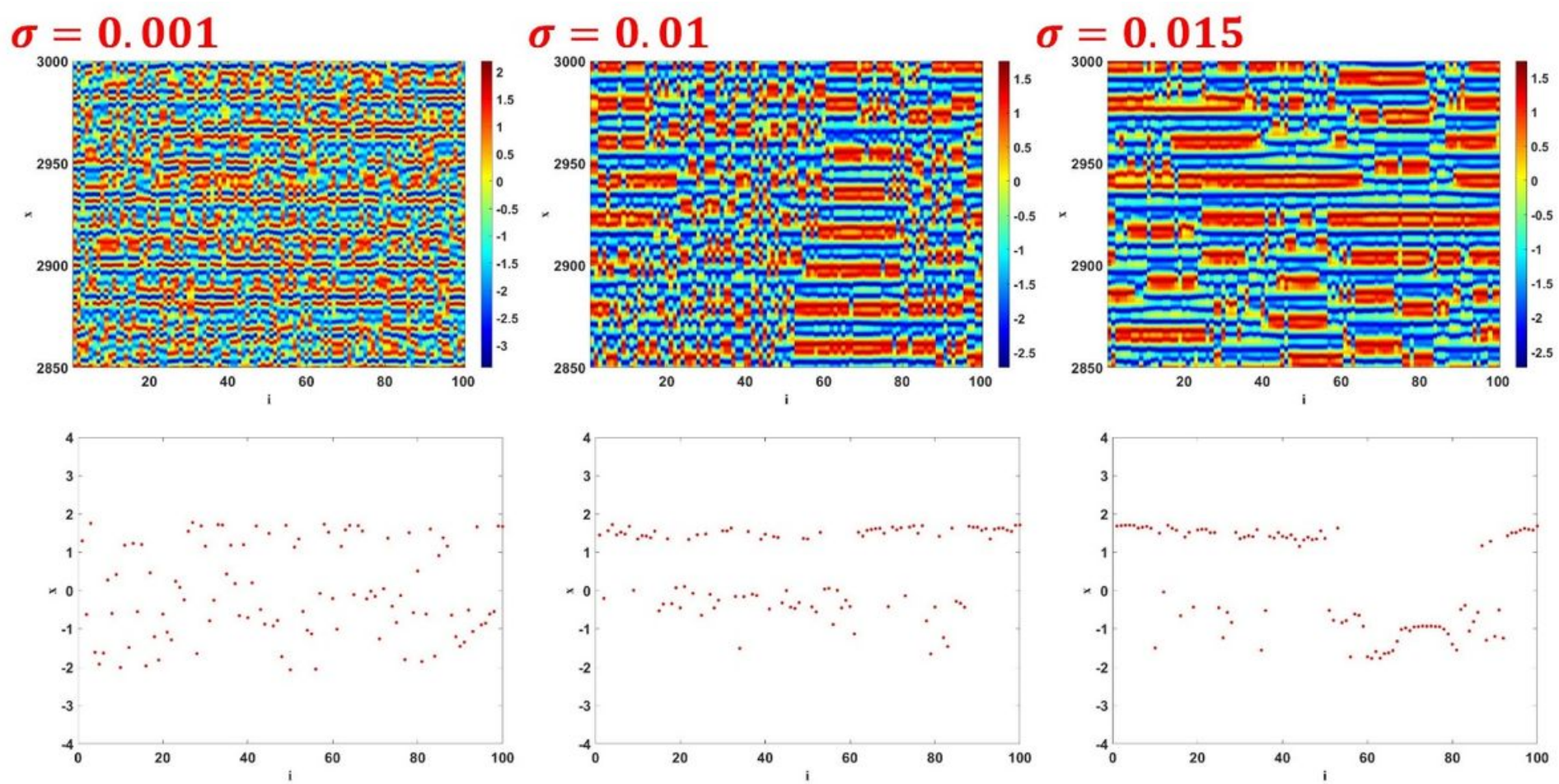

Figure 8

The collective behaviour of the network (1) for different values of the coupling coefficient $(\sigma)$ considering a periodic excitation. The plots confirm that the nodes are asynchronous.

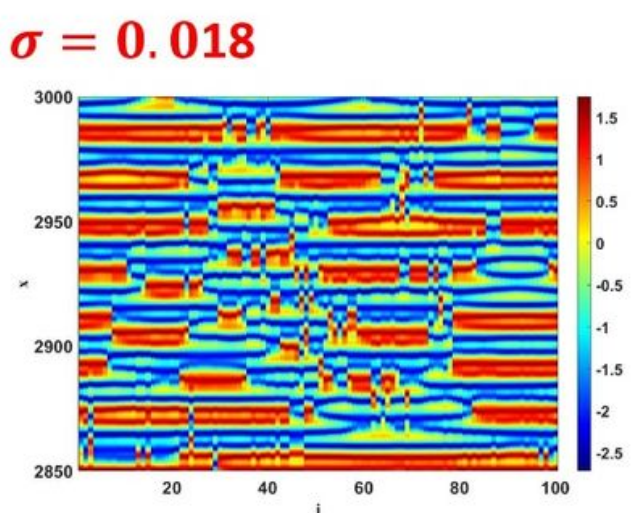

$$
\sigma=0.02
$$

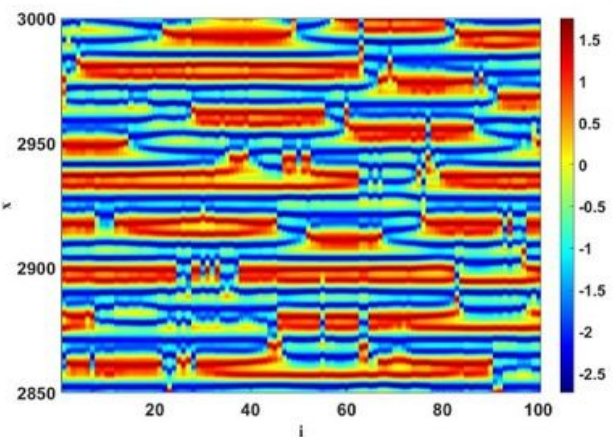

$$
\sigma=0.022
$$
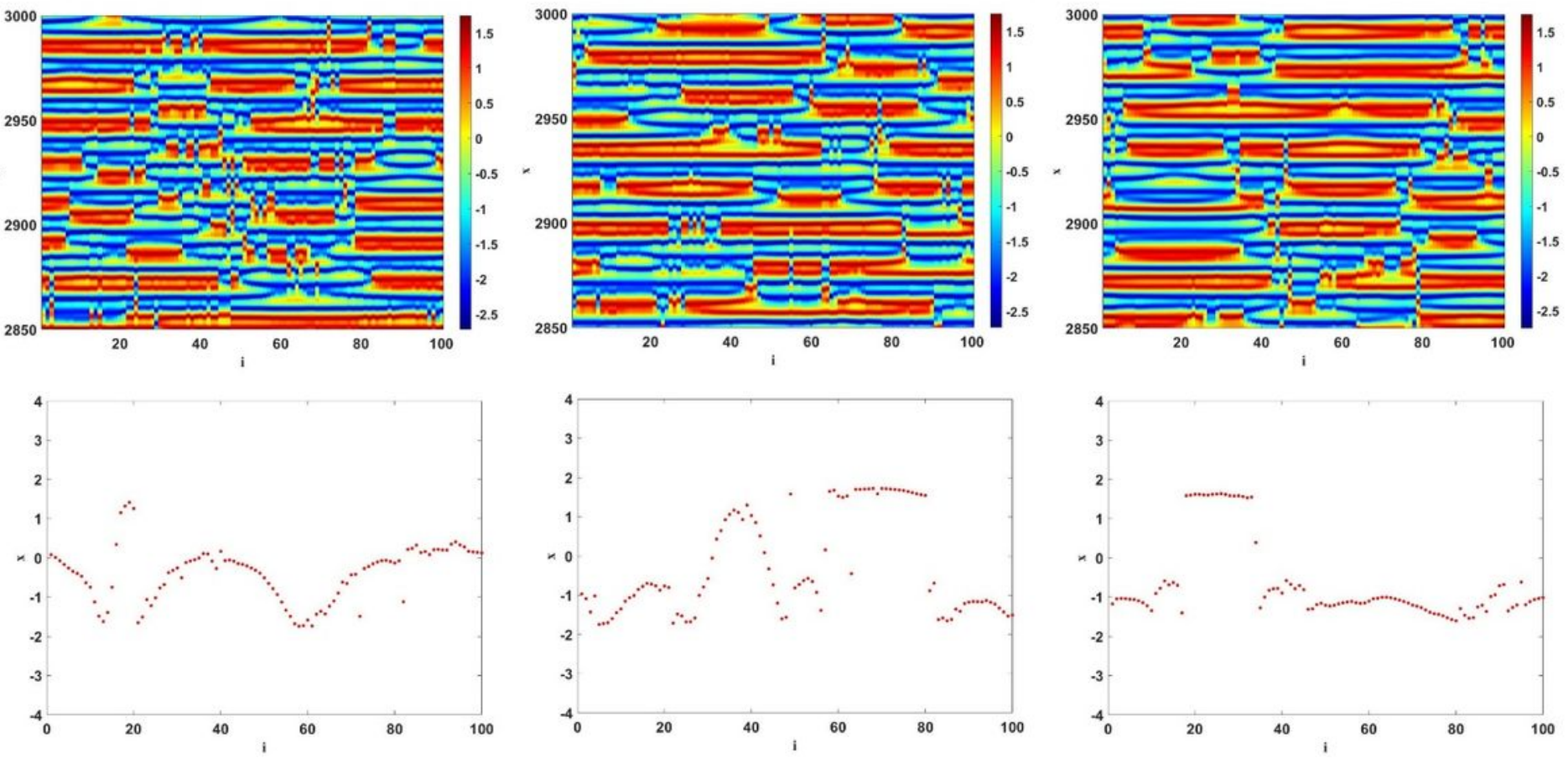

Figure 9 
The collective behaviour of the network (25) for different values of the coupling coefficient $(\sigma)$ considering a periodic excitation. The plots confirm that the nodes are showing both synchronous and asynchronous nodes confirming the existence of chimeras. 